\title{
Hepatic Oncostatin M Receptor $\beta$ Regulates Obesity-Induced Steatosis and Insulin Resistance
}

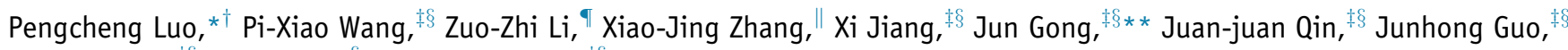
Xueyong Zhu, ${ }^{\ddagger \S}$ Sijun Yang, ${ }^{\S}$ and Hongliang $\mathrm{Li}^{\ddagger \S}$

From the Departments of Nephrology* and Cardiology, ${ }^{\ddagger}$ Renmin Hospital of Wuhan University, Wuhan; the Huangshi Central Hospital, ${ }^{\dagger}$ Hubei Polytechnic University, Huangshi; the Animal Experiment Center/Animal Biosafety Level-III Laboratory, ${ }^{\S}$ and the College of Life Sciences, ** Wuhan University, Wuhan; the Department of Cardiology, " Fuwai Hospital, National Center for Cardiovascular Diseases, Chinese Academy of Medical Sciences and Peking Union Medical College, Beijing; and the State Key Laboratory of Quality Research in Chinese Medicine, Institute of Chinese Medical Sciences, University of Macau, Macao, China

Accepted for publication

December 31, 2015.

Address correspondence to Hongliang Li, M.D., Ph.D., Department of Cardiology, Renmin Hospital of Wuhan University, Animal Experiment Center/Animal Biosafety LevelIII Laboratory, Collaborative Innovation Center of Model Animal, Cardiovascular Research Institute, Wuhan University, Luojia Mount Wuchang, Wuhan 430072, China. E-mail: lihl@whu.edu.cn.

\begin{abstract}
The liver is an essential insulin-responsive organ that is critical for maintaining glucose homeostasis and lipid metabolism. Oncostatin M receptor $\beta$ chain (OSMR $\beta$ ) is implicated in adipose tissue- and immune cellmediated metabolic regulation. However, the role of hepatocyte-derived OSMR $\beta$ in metabolic disorders remains unclear. Here, we report on the central role of OSMR $\beta$ in the protection against obesity and deregulation of glucose and lipids. We observed significantly varied expression levels of OSMR $\beta$ in hepatic tissues in both human samples and mouse models of nonalcoholic fatty liver disease. Mice lacking either whole-body or hepatic OSMR $\beta$ displayed exacerbated diet-induced insulin resistance, hepatic steatosis, and inflammation, both in diet-induced and genetically $(o b / o b)$ obese mice. These adverse effects were markedly attenuated by hepatocyte-specific overexpression of OSMRB. Mechanistically, we showed that OSMR $\beta$ phosphorylates and activates the Janus kinase 2 (JAK2)/STAT3 signaling pathway in the liver. More importantly, the liver-restricted overexpression of STAT3 rescued glucose tolerance and ameliorated hepatic steatosis and inflammation in OSMR $\beta$ knockout mice, whereas OSMR $\beta$ overexpression failed to protect against hepatic steatosis, insulin resistance, and hepatic inflammation in STAT3-deficient mice. Thus, activation of STAT3 is both sufficient and required to produce OSMR $\beta$-mediated beneficial effects. In conclusion, hepatic OSMR $\beta$ expression alleviates obesity-induced hepatic insulin resistance and steatosis through the activation of JAK2/STAT3 signaling cascades. (Am J Pathol 2016, 186: 1278-1292; http:// dx.doi.org/10.1016/j.ajpath.2015.12.028)
\end{abstract}

Nonalcoholic fatty liver disease (NAFLD) is the most prevalent liver disorder in the Western world and affects up to $30 \%$ of the general population and $75 \%$ to $100 \%$ of obese individuals. ${ }^{1,2}$ Most patients with NAFLD manifest a certain degree of hepatic insulin resistance (IR) and dyslipidemia, ${ }^{1}$ with obesity closely linked to the development of insulin resistance and diabetes mellitus. ${ }^{3}$ The ectopic accumulation of hepatic lipids is an early manifestation of NAFLD that inhibits the insulin receptor kinase, ${ }^{2}$ and increased serum concentrations of glucose and lipids may increase the affected individual's susceptibility to oxidative damage and inflammation. ${ }^{4}$ Insulin regulation involves an intricate relay of signaling cascades that include the phosphorylation and activation of insulin receptor substrates (IRSs) and downstream AKT and Forkhead box protein O1 (FOXO1). ${ }^{5,6}$ Defects in insulin-induced signaling cascades coupled with nutritional oversupply contribute to the deregulation of lipid and glucose metabolism. Unfortunately, current therapies for NAFLD are limited to weight loss, and effective drug therapies have not been developed. ${ }^{5}$ Thus, novel therapies for NAFLD are warranted.

Supported by the National Science Fund for Distinguished Young Scholars grant 81425005 (H.L.); the National Natural Science Foundation of China grants 81170086 and 81270184 (H.L.); National Science and Technology Support Project grants 2011BAI15B02, 2012BAI39B05, 2013YQ030923-05, 2014BAI02B01, and 2015BAI08B01 (H.L.); the Key Project of the National Natural Science Foundation grant 81330005 (H.L.); the National Basic Research Program China grant 2011CB503902 (H.L.), and Natural Science Foundation of Hubei Province grant 2013CFB259 (H.L.).

P.L., P.-X.W., and Z.-Z.L. contributed equally to this work.

Disclosures: None declared. 
The liver is an essential metabolic organ for glucose homeostasis and lipid metabolism, including lipogenesis, lipid uptake, and fatty acid $\beta$-oxidation. In the development of IR, growing evidence indicates the crucial role of hepatocytes instead of changes in circulating adipocytokines. For instance, our group and others have shown that mice presenting targeted overexpression of IRF3, IRF9, and STAT3 in the liver display ameliorated hepatic IR and liver-specific steatosis. ${ }^{7-9}$ Nevertheless, despite the increased prevalence and potentially severe consequences, the underlying mechanism of IR and hepatic steatosis are not completely understood.

Oncostatin M (OSM) was initially characterized from its antiproliferative effect on A375 melanoma and is a member of the gp130 [or IL-6/leukemia inhibitory factor receptor (LIFR)] cytokine family. ${ }^{10}$ The pathophysiologic functions of OSM in the liver are diverse. OSM was initially identified as a potent inducer of hepatocyte acute-phase protein responses. ${ }^{11}$ Studies have shown that OSM is also involved in iron metabolism regulation by transcriptional induction of hepatic hepcidin. ${ }^{12}$ Moreover, OSM may also help coordinate the development of liver differentiation and hematopoiesis. ${ }^{13,14}$ Notably, OSM engages a second receptor, either LIFR $\alpha$ or OSM receptor $\beta$ chain (OSMR $\beta$ ), before recruiting gp130. ${ }^{10} \mathrm{OSM} / \mathrm{gp} 130$ then initiates the activation of several transcription factors, including STAT3 and mitogen-activated protein kinase (MAPK), both of which are modulators of glucose regulation. ${ }^{9,15,16}$ Interestingly, OSM has been shown to up-regulate HepG2 low-density lipoprotein receptor expression, indicating a potential role in hepatic lipid metabolism, which is supported by the metabolic effect of OSM in Kupffer cells. ${ }^{10,17,18}$ Nevertheless, previous studies primarily focused on the role of OSM/ OSMR $\beta$ in adipocyte and liver immune cells, and the underlying functional role of hepatocyte-derived OSMR $\beta$ in the myriad interrelated disorders, including IR, dyslipidemia, hepatic inflammation, and steatosis, is unknown. ${ }^{17-20}$

Here, we found that NAFLD correlated with decreased expression levels of OSMR $\beta$ in hepatic tissues in both human and mouse models. More importantly, we showed that hepatocyte-derived OSMR $\beta$ provides protection against obesity-induced hepatic IR, inflammatory responses, and steatosis through the activation of Janus kinase 2 (JAK2)/ STAT3 in the liver. Furthermore, by genetically manipulating STAT3 in mice, we showed that STAT3 is both sufficient and required for OSMR $\beta$-mediated beneficial effects. Thus, targeting OSM/OSMR $\beta$ may improve hepatic lipid metabolism and insulin sensitivity.

\section{Materials and Methods}

\section{Animals and Diets}

All of the animal experiments were approved by the Animal Care and Use Committee of Renmin Hospital of Wuhan University. Liver-specific cyclization recombination enzyme
(Cre) transgenic mice [albumin (Alb)-Cre; stock no. 003574] and STAT3 conditional (floxed) mutant mice (STAT3 ${ }^{\text {flox/flox; }}$; stock no. 016923) were purchased from The Jackson Laboratory (Bar Harbor, ME). Full-length mouse OSMR $\beta$ cDNA was inserted after a pCAG-CAT promoter that expressed the $C A T$ gene, which was flanked by loxP sites. The pCAG-CATOSMR $\beta$ construct was microinjected into fertilized embryos (C57BL/6J background) to produce OSMR $\beta$-floxed mice. Subsequently, liver-specific OSMR $\beta$ transgenic (OSMR $\beta$-TG) mice were generated by crossing OSMR $\beta$-floxed mice with Alb-Cre mice, and liver-specific STAT3-knockout (KO) mice were produced by mating STAT3 ${ }^{\text {flox/flox }}$ mice with Alb-Cre mice. The liver-specific STAT3-TG mice were generated with similar procedures to those used to generate the OSMR $\beta$ TG mice, and the primers used for genotyping were as follows: CAG forward, 5'-CCCCCTGAACCTGAAACATA-3'; and Stat3C reverse, 5'-GCAATCTCCATTGGCTTCTC- $3^{\prime}$. Global OSMR $\beta$-KO mice were established with the clustered regularly interspaced short palindromic repeats (CRISPR)/Cas system, as previously described. ${ }^{21}$ Briefly, guide sequences of the target site for OSMR $\beta$ gene in the mouse genome were predicted by the online CRISPR design tool (http://crispr.mit. edu; last accessed December 3, 2015). After in vitro transcription, mouse zygotes were injected with a mixture of NLSflag-linker-Cas9 mRNA (no. 44758; Addgene, Cambridge, MA) and OSMR $\beta$-single guide RNA. DNA sequences for genotyping primers are as follows: forward, $5^{\prime}$-ATTGCCCAGCAAGTTCTTTG-3' ${ }^{\prime}$, and reverse, $5^{\prime}$-CACACAGGGATGCAATTGTT- $3^{\prime}$. OSMR $\beta$-KO mice were crossed with STAT3-TG mice to obtain OSMR $\beta$-KO/STAT3-TG (OKST) mice. OSMR $\beta$-TG/STAT3-KO (OTSK) mice were then produced by crossing OSMR $\beta$-TG mice with STAT3-KO mice. The $o b / o b$ mice were purchased from The Jackson Laboratory (stock no. 000632). Eight-week-old male mice were used in this study, and they were fed either normal chow (protein, 18.3\%; fat, 10.2\%; carbohydrates, 71.5\%; D12450B; Research Diets, Inc., New Brunswick, NJ) or a high-fat diet (HFD; protein, 18.1\%; fat, 61.6\%; carbohydrates, 20.3\%; D12492; Research Diets, Inc.) ad libitum for up to 24 weeks. All of the animals were housed in an environment under a 12-hour light/dark cycle at a fixed temperature and humidity and given ad libitum access to food and water. Mouse body weights and fasting blood glucose concentrations were monitored every 4 weeks, and the food intake amounts were monitored weekly. The experimenters (X.-J.Z., X.J., J.Go., J.-j.Q., J.Gu., and X.Z.) were blinded to the genotypes of the animals.

\section{Tissue Preparation}

After anesthetization with $3 \%$ pentobarbital sodium, the animals were euthanized, and the livers were immediately removed and weighed. Each liver was divided into two parts, with one part immediately frozen in liquid nitrogen at $-80^{\circ} \mathrm{C}$ and the other part fixed in $10 \%$ formalin or frozen with Tissue-Tek OCT Compound (Tokyo, Japan) in dry ice 
before embedding. Liver tissues sections were prepared and stained with hematoxylin and eosin $(5 \mu \mathrm{m}$ per section) or Oil Red O stain $(4 \mu \mathrm{m})$. The sections were then counterstained with Mayer hematoxylin after destaining in $60 \%$ isopropanol.

\section{Metabolic Studies and Serum Cytokine Analyses}

For the glucose tolerance tests (GTTs) and insulin tolerance tests (ITTs), $1 \mathrm{~g} / \mathrm{kg}$ glucose (Sigma-Aldrich Co., St. Louis, $\mathrm{MO}$ ) or $0.75 \mathrm{U} / \mathrm{kg}$ insulin (Novolin R; Novo Nordisk Co., Bagsvaerd, Denmark), respectively, were intraperitoneally injected into mice. Blood glucose concentrations were detected with a glucometer (One Touch Ultra Easy; LifeScan, Inc., Milpitas, CA) after a 6-hour fast before injection and 15, 30, 60 , and 120 minutes after glucose or insulin injection. Serum fasting insulin was examined by enzyme-linked immunosorbent assay (Millipore, Billerica, MA). The homeostasis model assessment of the IR (HOMA-IR) index was calculated as HOMA-IR $=[\mathrm{FBG}(\mathrm{mmol} / \mathrm{L}) \times$ FINS $(\mathrm{mIU} / \mathrm{L})] / 22.5$.

Hepatic triglyceride, total cholesterol (TC), and nonesterified fatty acid (NEFA) concentrations were determined with commercial kits (Wako Chemicals, Richmond VA). $\beta$-Hydroxybutyrate serum concentrations were examined with commercial kits (Abcam, Cambridge, UK). Serum cytokines IL-1 $\beta$, IL-6, IL-4, tumor necrosis factor (TNF)- $\alpha$, monocyte chemoattractant protein-1 (MCP1), IL-10, leptin, resistin, and adiponectin were assessed via enzyme-linked immunosorbent assay (R\&D Systems, Minneapolis, MN; MBL International, Woburn, MA; RayBiotech, Norcross, GA; Invitrogen, Carlsbad, CA; PeproTech, Rocky Hill, NJ).

\section{Assessment of Liver Function}

The concentrations of alanine transaminase and aspartate transaminase in the serum were measured with an ADVIA 2400 Chemistry System analyzer (Siemens, Tarrytown, NY) according to the manufacturer's instructions.

\section{Quantitative Real-Time PCR}

Total RNA was extracted from liver tissue with the use of TRIzol reagent (Invitrogen) according to the manufacturer's protocol and was reverse-transcribed into cDNA with the use of a Transcriptor First-Strand cDNA Synthesis Kit (Roche, Indianapolis, IN). A LightCycler 480 SYBR Green 1 Master Mix (Roche) and LightCycler 480 QPCR System (Roche) were used to perform the quantitative real-time PCR analysis. The PCR conditions were as follows: $95^{\circ} \mathrm{C}$ for 10 minutes; 40 cycles of $95^{\circ} \mathrm{C}$ for 10 seconds, $60^{\circ} \mathrm{C}$ for 10 seconds, and $72^{\circ} \mathrm{C}$ for 20 seconds; and a final extension at $72^{\circ} \mathrm{C}$ for 10 minutes. The relative quantity of the mRNAs was calculated by normalization to the quantity of GAPDH mRNA. The primer pairs are listed in Table 1.

Table 1 Primers for Real-Time PCR Detection

\begin{tabular}{|c|c|c|}
\hline Gene & Forward primer & Reverse primer \\
\hline GAPDH & $5^{\prime}-$ ACTCCACTCACGGCAAATTC- $3^{\prime}$ & $5^{\prime}-$ TCTCCATGGTGGTGAAGACA-3' \\
\hline OSMRB & 5'-TTTGTGCGCTGTGCAAGTGC-3' & 5'-TGCGTCTTCCATTCTCCGACC-3' \\
\hline$A B C G 1$ & 5'-TGAACCCGTTTCTTTGGCACCG-3' & $5^{\prime}$-AGTCCCGCATGATGCTGAGGAA- 3' \\
\hline CYP7A1 & 5'-TCAAAGAGCGCTGTCTGGGTCA-3' & 5'-TTTCCCGGGCTTTATGTGCGGT-3' \\
\hline SREBP1C & $5^{\prime}$-CACTTCTGGAGACATCGCAAAC-3' & $5^{\prime}-$ ATGGTAGACAACAGCCGCATC- $3^{\prime}$ \\
\hline$F A B P 1$ & 5'-TGGTCCGCAATGAGTTCACCCT-3' & 5'-CCAGCTTGACGACTGCCTTGACTT-3' \\
\hline FATP1 & 5'-TGCACAGCAGGTACTACCGCAT-3' & $5^{\prime}-\mathrm{TGCGCAGTACCACCGTCAAC-3^{ \prime }}$ \\
\hline PPARG & $5^{\prime}$-АTTCTGGCCCACCAACTTCGG-3' & 5'-TGGAAGCCTGATGCTTTATCCCCA-3' \\
\hline$A C C A$ & $5^{\prime}$-GGCCAGTGCTATGCTGAGAT-3' & $5^{\prime}$-AGGGTCAAGTGCTGCTCCA-3' \\
\hline$S C D 1$ & $5^{\prime}$-тСТTCСTTATCATTGCCAACACCA-3' & $5^{\prime}$-GCGTTGAGCACCAGAGTGTATCG-3' \\
\hline$\angle C A D$ & $5^{\prime}-$ GGAGTAAGAACGAACGCCAA- $3^{\prime}$ & $5^{\prime}-$ GCCACGACGATCACGAGAT-3' \\
\hline ACOX1 & $5^{\prime}$-CGGAAGATACATAAAGGAGACC-3' & $5^{\prime}-\mathrm{AAGTAGGACACCATACCACCC}-3^{\prime}$ \\
\hline CPT1A & $5^{\prime}$-AGGACCCTGAGGCATCTATT-3' & $5^{\prime}-\mathrm{ATGACCTCCTGGCATTCTCC-3^{ \prime }}$ \\
\hline PPARA & $5^{\prime}$-TATTCGGCTGAAGCTGGTGTAC- $3^{\prime}$ & 5'-CTGGCATTTGTTCCGGTTCT-3' \\
\hline$I L 1 B$ & $5^{\prime}$-CCGTGGACCTTCCAGGATGA-3' & $5^{\prime}$-GGGAACGTCACACACCAGCA-3' \\
\hline IL6 & 5'-AGTTGCCTTCTTGGGACTGA-3' & $5^{\prime}-$ TCCACGATTTCCCAGAGAAC- $3^{\prime}$ \\
\hline TNFA & 5'-CATCTTCTCAAAATTCGAGTGACAA-3' & $5^{\prime}-$ TGGGAGTAGACAAGGTACAACCC $-3^{\prime}$ \\
\hline$M C P 1$ & 5'-TAAAAACCTGGATCGGAACCAAA- $3^{\prime}$ & 5'-GCATTAGCTTCAGATTTACGGGT-3' \\
\hline INOS & 5'-TGCGCCTTTGCTCATGACATCGA-3' & $5^{\prime}$-ATGGATGCTGCTGAGGGCTCTGTT-3' \\
\hline IL10 & $5^{\prime}$-CCAAGCCTTATCGGAAATGA-3' & 5'-TTTTCACAGGGGAGAAATCG-3' \\
\hline
\end{tabular}




\section{Western Blot Analysis}

Antibodies against OSMR $\beta$ (sc30010) were purchased from Santa Cruz Biotechnology (Dallas, TX). The antibody against p-IRS1 (09-432) was purchased from Millipore (Billerica, MA). Antibodies to IRS1 (2382), p-Akt (4060), Akt (4691), p-GSK3 $\beta$ (9322), GSK3 $\beta$ (9315), p-FOXO1 (9461), FOXO1 (2880), IKK $\beta$ (2370), p-P65 (3033), P65

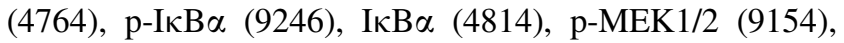
MEK1/2 (9122), p-ERK1/2 (4370), ERK1/2 (4695), p-P38 (4511), P38 (9212), p-JNK (4668), JNK (9258), p-JAK2 (3776), JAK2 (3230), p-STAT3 (9145), and GAPDH (2118) were obtained from Cell Signaling Technology (Boston, MA). The STAT3 antibody (BS1336) was obtained from Bioworld Technology (Harrogate, UK), and antibody against p-IKK $\beta$ (Ab5915) was ordered from Abcam.

The livers or cultured cells were lyzed in lysis buffer, and $50 \mu \mathrm{g}$ of extracted protein was separated on $8 \%$ to $12 \%$ SDS-PAGE gels and transferred to polyvinylidene difluoride membranes (Millipore, Bedford, MA). The membranes were blocked in tris-buffered saline and Tween 20 that contained 5\% skim milk powder for 1 hour at room temperature and then incubated in primary antibodies at $4{ }^{\circ} \mathrm{C}$ overnight. Next, the membranes were washed and then incubated with secondary antibodies for 1 hour at room temperature. A ChemiDoc MP Imaging System (Bio-Rad, Hercules, CA) was used for signal detection. Protein expression levels were quantified with Image Lab Software version 5.1 (Bio-Rad) and normalized to the loading control GAPDH.

\section{Cell Culture and in Vitro Model of Hepatic Steatosis}

After anesthetization, mouse livers were perfused with perfusion buffer $(0.9 \%$ saline) in situ through the superior vena cava, followed by digestion buffer (D Hanks' solution supplemented with $0.05 \%$ trypsin 25200; Gibco, Carlsbad, CA). The cell suspension was filtered and then centrifuged at $50 \times g$ for 5 minutes. The harvested hepatocytes were then resuspended in Dulbecco's modified Eagle's medium (15\% fetal bovine serum, $5 \mu \mathrm{g} / \mathrm{mL}$ insulin, $100 \mathrm{U} / \mathrm{mL}$ penicillin, $100 \mathrm{U} / \mathrm{mL}$ streptomycin) before seeding on collagen-coated dishes (Sigma-Aldrich Co.). After cultivation for 24 hours $\left(5 \% \mathrm{CO}_{2}, 37^{\circ} \mathrm{C}\right)$, the medium was replaced with fresh fetal bovine serum-free media, and the cells were then incubated for another 24 hours before adenovirus administration. To establish an in vitro model of hepatic steatosis ${ }^{22}$ palmitate $(0.25 \mathrm{mmol} / \mathrm{L}$; Sigma-Aldrich Co. $)$ was added to the medium of cultured hepatocytes and incubated for an additional 24 hours.

\section{Recombinant Adenovirus Vector Production and Transfection}

Adenoviruses carrying sequences encoding mouse OSMR $\beta$ (AdOSMR $\beta$ ) and a shRNA targeting OSMR $\beta$ (AdshOSMR $\beta$ ) were constructed as previously described. ${ }^{23}$ Similar adenoviral vectors encoding the GFP gene (AdGFP) or scrambled shRNA (AdshRNA) were used as controls. The adenoviruses were transfected into cultured hepatocytes at a multiplicity of infection of 100 for 48 hours. For in vivo studies, we injected appropriate adenoviruses $\left(5 \times 10^{9}\right.$ plaque-forming units) into the jugular vein as previously described. ${ }^{23}$ Four weeks after adenoviral injection, the mice were euthanized, and their tissues were harvested.

\section{Human Liver Tissue Samples}

Samples of human NAFLD were collected from NAFLD patients undergoing liver biopsy to diagnose fatty liver. Control samples were obtained from normal liver donors who died from accidents, but their livers were not suitable for transplantation for nonhepatic reasons. Clinical and histologic characteristics of these samples are provided in Supplemental Tables S1 and S2. Written informed consent was obtained from the families of the prospective liver donors. All procedures that involved human samples were approved by the Renmin Hospital of Wuhan University Review Board, Wuhan, China.

\section{Statistical Analysis}

All data are expressed as the means \pm SD. Differences among groups were determined with a one-way analysis of variance with a subsequent least squares difference test (assuming equal variances) or Tamhane's T2 test (without the assumption of equal variances). Comparisons between two groups were performed with the two-tailed Student's $t$-test. $P<0.05$ was considered significant. The data analysis and imaging studies were performed in a blinded manner (X.-J.Z., X.J., J.Go., J.-j.Q., and J.Gu.).

\section{Results}

OSMR $\beta$ Protein Expression Is Decreased in Mice with Diet-Induced and Genetic Obesity

To determine the involvement of OSMR $\beta$ in humanpathologic processes, we first investigated OSMR $\beta$ expression levels in the liver samples of patients with NAFLD. Interestingly, OSMR $\beta$ expression in the NAFLD livers was reduced to approximately $35.7 \%$ that of the normal controls (Figure 1A). OSMR $\beta$ protein expression was also reduced in a cell model of steatosis induced by palmitate treatment (Figure 1B). ${ }^{22}$ Next, we examined hepatic OSMR $\beta$ expression in HFD-induced and genetic $(o b / o b)$ obesity models, both of which induce several features of human NAFLD, including lipid accumulation, inflammation, and hepatic steatosis. ${ }^{24}$ Mice were fed a normal chow diet or HFD for 24 weeks. The HFD administration for 8 weeks led to significantly up-regulated protein expression of OSMR $\beta$, whereas hepatic OSMR $\beta$ protein 

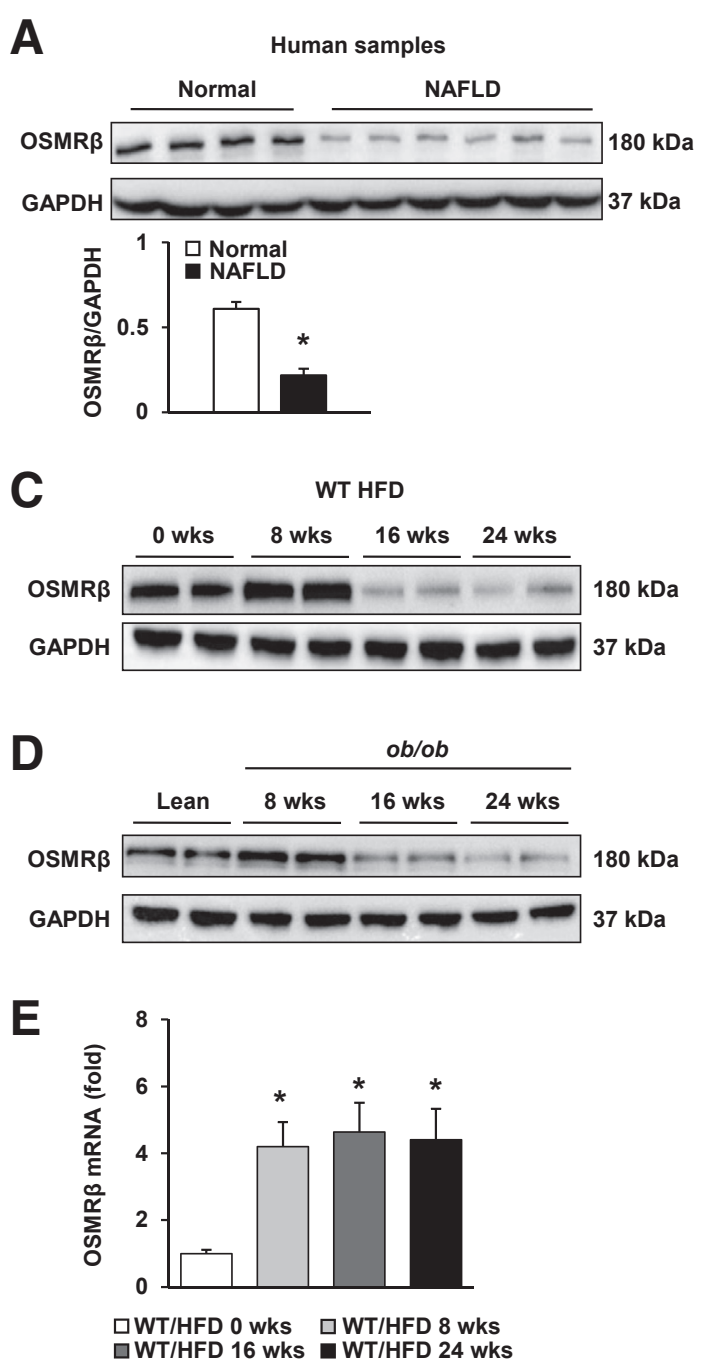
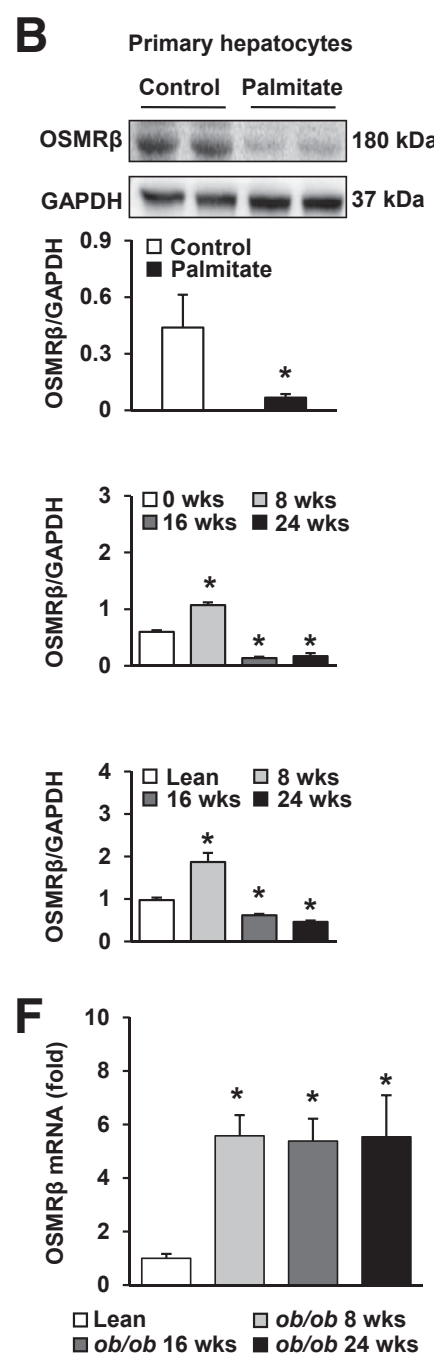

Figure 1 OSMR $\beta$ protein expression is reduced in the livers of obese mice and NAFLD patients. A: Total liver homogenates were harvested from human control or NAFLD samples and subjected to immunoblotting. B: Primary hepatocytes were challenged with $0.25 \mathrm{mmol} / \mathrm{L}$ palmitate, and protein levels of OSMR $\beta$ was detected via immunoblotting. C and D: Mouse liver homogenates were obtained at the indicated times. Protein expression and quantification of OSMR $\beta$. A-D: GAPDH was used as a loading control. $\mathbf{E}$ and $\mathbf{F}$ : The mRNA expressions of OSMR $\beta$ in the liver of WT mice fed an HFD (E) or $o b / o b$ mice and lean controls (F). Data are expressed as means \pm SD. $n=4$ for normal and $n=6$ for NAFLD (A); $n=4$ independent experiments (B); $n=4$ per time point (C and D). ${ }^{*} P<0.05$ versus control. GAPDH, glyceraldehyde-3-phosphate dehydrogenase; HFD, high-fat diet; NAFLD, nonalcoholic fatty liver disease; OSMR $\beta$, OSM receptor $\beta$ chain; WT, wild-type. was dramatically down-regulated in mice challenged with HFD for 16 or 24 weeks compared with that of the control group (Figure 1C). Interestingly, mRNA levels of hepatic OSMR $\beta$ remained at a higher level between 8 and 24 weeks after HFD administration than for NC controls (Figure 1E). Moreover, a similar expression profile of hepatic OSMR $\beta$ expression was observed in the $o b / o b$ mice (Figure 1, D and F). Collectively, this reduction in hepatic OSMR $\beta$ protein expression indicates potential biological significance in both mice and humans.

\section{OSMR $\beta$ Deletion Exacerbates Obesity and Metabolic Dysfunction}

Because hepatic OSMR $\beta$ was observed to decrease in obese mice and NAFLD patients, we next investigated the biological effect of this reduction on metabolic disorders. To address this issue, global OSMR $\beta$-KO mice were established with the clustered regularly interspaced short palindromic repeats (CRISPR)/Cas system (Supplemental Figure S1, $\mathrm{A}-\mathrm{C}$ ), and OSMR $\beta$ ablation was confirmed with direct sequencing of PCR products (Supplemental Figure S1D) and immunoblotting (Supplemental Figure S1E). Intriguingly, although body weight was comparable between OSMR $\beta-\mathrm{KO}$ and wild-type (WT) mice fed an NC diet, HFD-induced obesity was more severe in the OSMR $\beta$-KO mice than in their WT littermates (Figure 2A). Functionally, glucose metabolic disorders were also exacerbated on OSMR $\beta$ ablation as evidenced by significantly higher fasting blood glucose (Figure 2B) and insulin concentrations (Figure 2C) in the OSMR $\beta-\mathrm{KO}$ mice than in the WT controls.

In addition, OSMR $\beta$-KO mice also exhibited a higher HOMA-IR index than that of the WT mice (Figure 2D). We then performed i.p. GTTs and i.p. ITTs in which OSMR $\beta$ KO mice exhibited defective insulin sensitivity compared with WT mice (Figure 2, E and F).

In response to insulin stimulation, IRS1 is recruited to the activated insulin receptor, which in turn phosphorylates and activates IRS1; the subsequent AKT/GSK3ß/FOXO1 phosphorylation cascade plays a central role in metabolic homeostasis. Immunoblotting showed that insulin-induced phosphorylation of IRS1, AKT, GSK3 $\beta$, and FOXO1 in OSMR $\beta$-KO mice was decreased compared with that of the WT controls, indicating impaired insulin regulation 
A

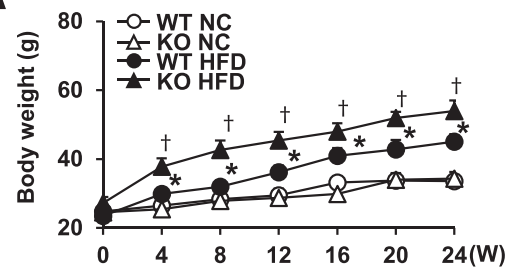

C

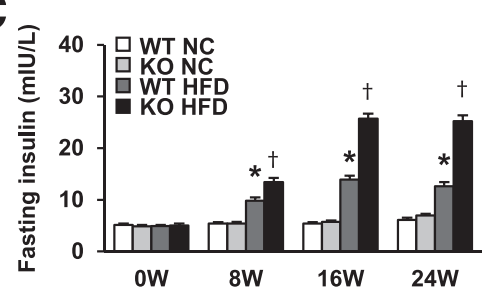

E

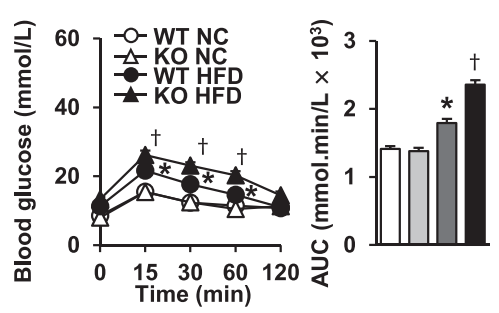

G

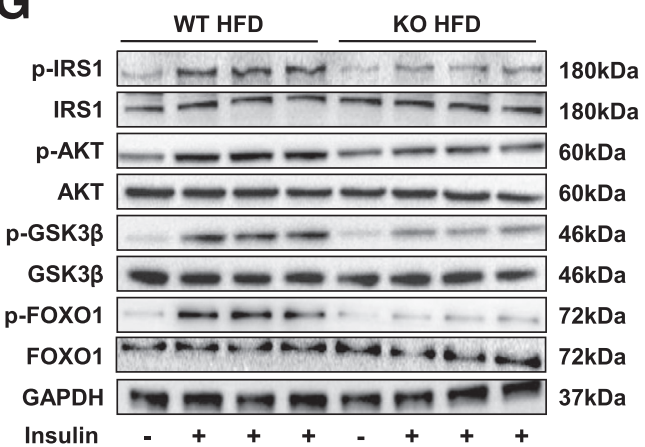

B

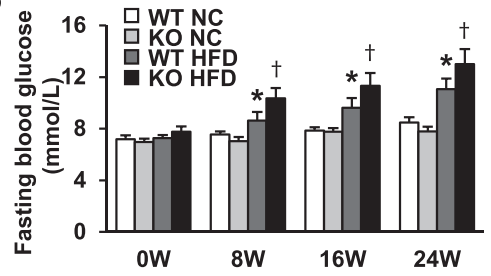

D

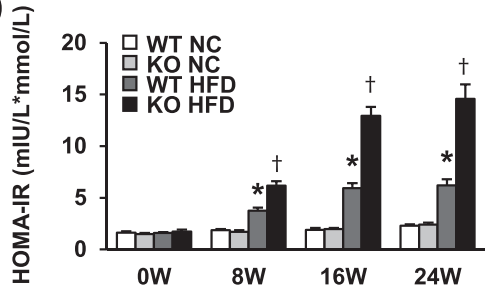

$\mathbf{F}$

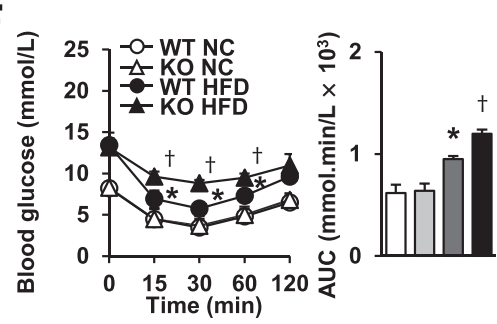

Figure 2 Deletion of OSMR $\beta$ aggravates obesity and insulin resistance. A: Body weight analysis of the WT and OSMR $\beta$-KO mice on the 24-week NC diet or HFD. B and C: Fasting serum glucose (B) and insulin (C) concentrations were assessed at the indicated time points. D: HOMA-IR index was calculated as [FBG $(\mathrm{mmol} / \mathrm{L}) \times$ FINS (mIU/L)]/22.5. E and F: OSMR $\beta-K 0$ mice display aggravated insulin resistance, and IPGTT $(1 \mathrm{~g} / \mathrm{kg}$ body weight) (E) and IPITT ( $0.75 \mathrm{U} / \mathrm{kg}$ body weight) (F) were performed on the WT and KO mice in both the NC and HFD groups. The corresponding AUC of the blood glucose concentrations in each group was calculated. G: Mouse liver homogenates were obtained from mice fed a HFD for 24 weeks, deprived of food overnight, and intraperitoneally injected with $0.75 \mathrm{U}$ insulin $/ \mathrm{kg}$ body weight for 10 minutes. Expression and quantification of the indicated proteins. Data are expressed as means \pm SD. $n=14$ to 21 per group $(\mathbf{A}) ; n=10$ to 12 per group ( $\mathbf{B}$ and $\mathbf{C}$ ); $n=10$ to 12 per group at each time point (D); $n=10$ to 14 per group at each time point (E and $\mathbf{F}$ ); $n=3$ independent experiments (G). GAPDH was used as a loading control. ${ }^{*} P<0.05$ versus the WT NC mice; ${ }^{\dagger} P<0.05$ versus WT HFD mice. AUC, area under the curve; GAPDH, glyceraldehyde-3-phosphate dehydrogenase; HFD, high-fat diet; HOMA-IR, homeostasis model assessment of insulin resistance; IPGTT, i.p. glucose tolerance test; IPITT, i.p. insulin tolerance test; $\mathrm{KO}$, knockout; $\mathrm{NC}$, normal chow; OSMR $\beta$, OSM receptor $\beta$ chain; WT, wildtype.

(Figure 2G). Considering that the global OSMR $\beta$-KO mice were used, we next examined its impacts on AKT activation in adipose tissue and skeletal muscle. On insulin stimulation, OSMR $\beta$-KO mice displayed significantly reduced expression level of phosphorylated AKT compared with that of WT mice in both adipose tissue and skeletal muscle (Supplemental Figure S2A).

Under pathophysiologic conditions, hepatic OSMR $\beta$ expression levels were reduced after HFD-induced obesity (Figure 1B); therefore, we next determined whether a liverspecific reduction of OSMR $\beta$ expression exerted a similar deleterious effect on IR. To this end, we used adenovirusmediated gene transfer, a well-established method that acutely delivers the gene of interest to the liver by jugular injection. ${ }^{7,8}$ Injections of adenoviruses carrying sequences encoding a shRNA targeting OSMR $\beta$ (AdshOSMR $\beta$ ) were administered to mice fed an HFD for 20 weeks. Four weeks later, OSMR $\beta$ expression diminished to approximately $34.2 \%$ that of adenoviral vectors encoding scrambled shRNA
(AdshRNA) controls in hepatic tissues (Supplemental Figure S3A). Although the body weight was comparable (Supplemental Figure S3B), this reduction in OSMR $\beta$ expression levels resulted in significantly elevated fasting serum glucose and insulin concentrations compared with that of the control animals (Supplemental Figure S3C). The i.p. GTT and ITT results indicated IR was further exacerbated in AdshOSMR $\beta$-treated mice (Supplemental Figure S3, D and E). Overall, these data show that OSMR $\beta$ ablation exacerbates HFD-induced IR.

\section{Hepatic OSMR $\beta$ Overexpression Improves IR}

Subsequently, we proposed that if the adverse reduction of hepatic OSMR $\beta$ expression was counteracted, then HFD-induced glucose metabolic disorders may show an improvement. Thus, we specifically overexpressed OSMR $\beta$ in hepatocytes by crossing OSMR $\beta^{\text {flox/flox }}$ mice with mice carrying a Cre recombinase driven by a 
A
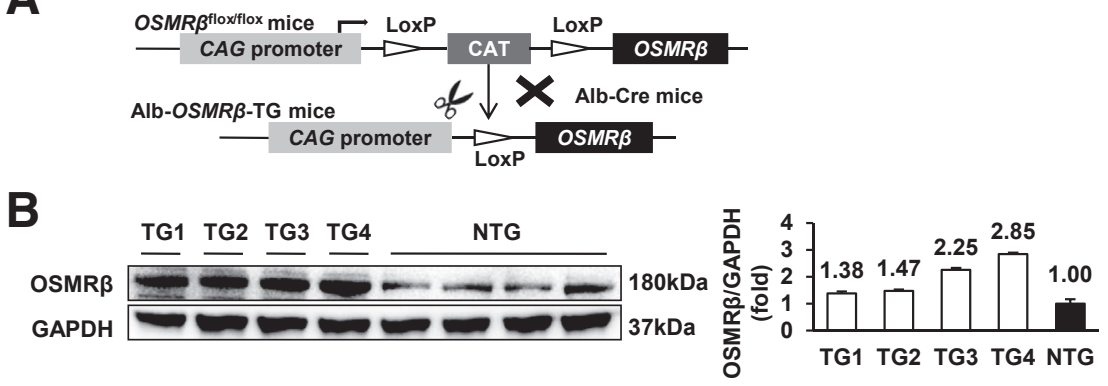
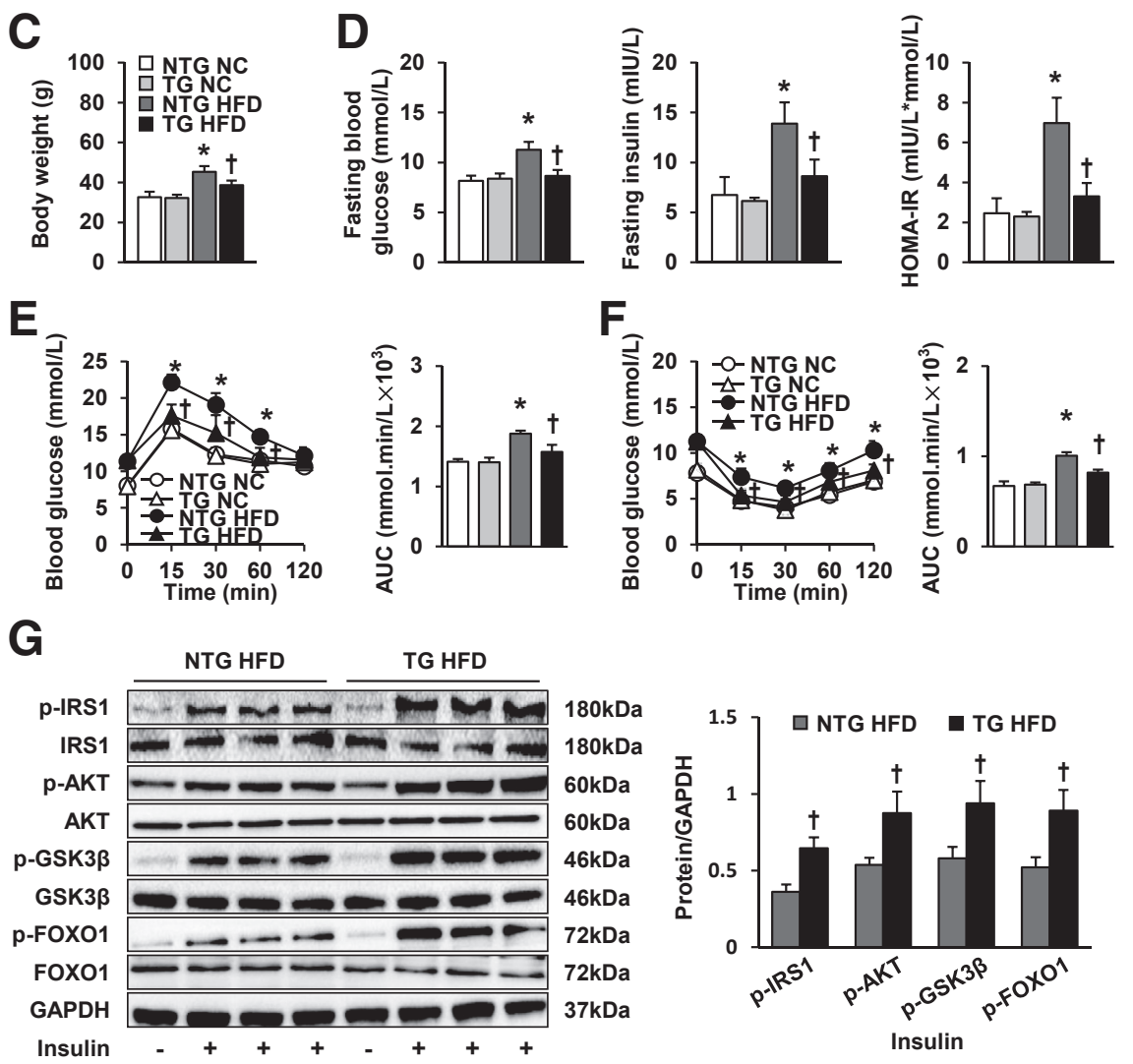

Figure 3 OSMR $\beta$ overexpression in hepatocytes alleviates hepatic insulin resistance. A: Schematic workflow of the establishment of a liver-specific OSMR $\beta$-overexpressing TG mouse strain. B: Immunoblotting of hepatic OSMR $\beta$ expression in OSMR $\beta$-TG mice. GAPDH was used as a loading control. C: Body weight analysis of NTG and OSMRß-TG mice after 24-week NC diet or HFD. D: Ameliorated glucose homeostasis in the OSMR $\beta$-TG mice. Fasting serum glucose and insulin concentrations were detected at the indicated time points. HOMA-IR was also calculated. $\mathbf{E}$ and $\mathbf{F}$ : Glucose tolerance was assessed by IPGTT (E) and IPITT (F). G: Expression and quantification of the indicated proteins in the IRS1/AKT signaling pathway. GAPDH was used as a loading control. Data are expressed as means \pm SD. $n=10$ to 14 per group (D); $n=10$ to 14 per group at each time point ( $\mathbf{E}$ and $\mathbf{F}) ; n=3$ independent experiments $(\mathbf{G}) .{ }^{*} P<0.05$ versus the NTG NC mice; ${ }^{\dagger} P<0.05$ versus NTG HFD mice. Alb, albumin; AUC, area under the curve; Cre, cyclization recombination enzyme; GAPDH, glyceraldehyde-3phosphate dehydrogenase; HFD, high-fat diet; HOMA-IR, homeostasis model assessment of insulin resistance; IPGTT, i.p. glucose tolerance test; IPITT, i.p. insulin tolerance test; NC, normal chow; NTG, nontransgenic; OSMR $\beta$, OSM receptor $\beta$ chain; TG, transgenic. hepatocyte-specific Alb promoter (Figure 3A). Four transgenic mouse lines, TG1 to TG4, were established, and TG4 (hereafter referred to as OSMR $\beta$-TG) mice were used for further experiments because TG4 displayed the highest OSMR $\beta$ expression in the liver (Figure 3B). Compared with non-TG mice, OSMR $\beta$-TG mice had significantly lower body weights (Figure 3C) and attenuated IR characterized by lower fasting serum glucose and insulin concentrations and a lower HOMA-IR index (Figure 3D). Glucose tolerance and insulin sensitivity were also improved on OSMR $\beta$ overexpression (Figure 3, E and F). Furthermore, insulin-induced phosphorylation of IRS1, AKT, GSK3 $\beta$, and FOXO1 was also increased in the OSMR $\beta$-TG mice, indicating preserved activation of the insulin cascade (Figure 3G). Intriguingly, hepatic-specific overexpression of OSMR $\beta$ also sufficiently enhanced insulin-induced phosphorylation of AKT in adipose tissue and skeletal muscle (Supplemental Figure S2B). Thus, these results revealed that hepatic OSMR $\beta$ is required for maintenance of normal glucose and insulin regulation in diet-induced obesity.

\section{OSMR $\beta$ Deletion Aggravates Hepatic Steatosis and Inflammatory Response}

Lipid accumulation in the liver, also known as hepatic steatosis, is strongly associated with hepatic IR, ${ }^{1}$ which prompted us to further investigate the impact of genetic manipulations to OSMR $\beta$ on liver morphology and lipid metabolism. After 24 weeks on the HFD, both global and liver-restricted OSMR $\beta$ ablation resulted in heavier livers than in the control mice (Figure $4 \mathrm{~A}$ and Supplemental Figure S3F). Conversely, liver weight remained low on OSMR $\beta$ overexpression in hepatocytes compared with that of non-TG mice when fed HFD (Figure 4A) and was associated with improved hepatic steatosis, which was 


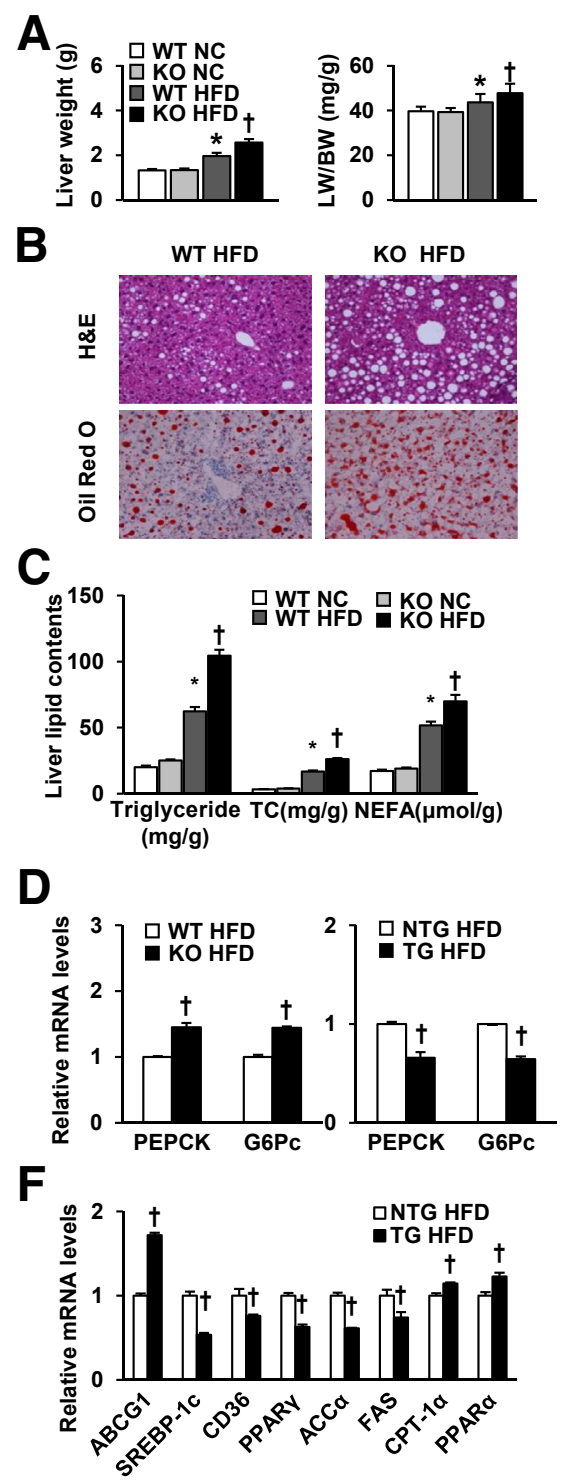

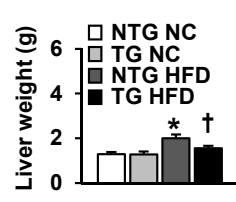
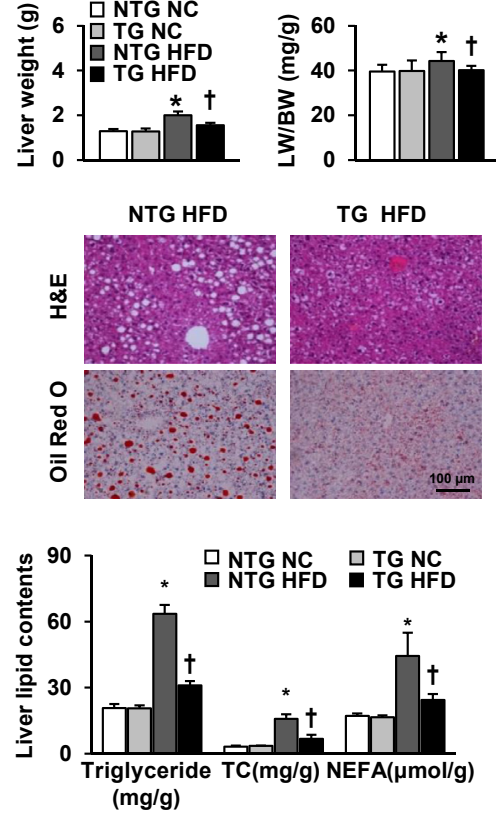

E

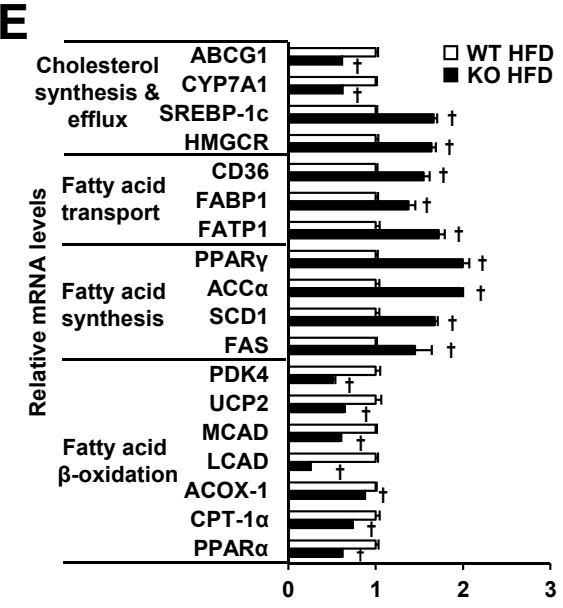

Figure 4 OSMR $\beta$ inhibits obesity-induced hepatic steatosis. A: Analysis of LW/BW in the indicated mice strains. B: Representative H\&E or 0il Red 0 staining of liver sections obtained from the indicated mice strains at 24 weeks of HFD feeding. C: Levels of liver triglycerides, TC, and NEFAs were examined in the WT and KO mice, NTG and OSMRB-TG mice on the HFD. D: Quantification of mRNA levels of PEPCK and G6Pc in the experimental conditions described in panels $\mathbf{A}-\mathbf{C}$. $\mathbf{E}$ and F: mRNA levels of genes related to lipid metabolism were detected via real-time PCR. Data are expressed as means \pm SD. $n=8$ to 10 per group (A); $n=8$ to 10 per group (C); $n=6$ to 8 per group (E and $\mathbf{F})$. ${ }^{*} P<0.05$ versus WT or NTG NC group; ${ }^{\dagger} P<0.05$ versus WT or NTG HFD group. G6PC, glucose-6-phosphatase; HFD, high-fat diet; $\mathrm{H} \& \mathrm{E}$, hematoxylin and eosin; KO, knockout; LW/ $\mathrm{BW}$, ratio of liver weight to body weight; NC, normal chow; NEFA, nonesterified fatty acid; NTG, nontransgenic; OSMR $\beta$, OSM receptor $\beta$ chain; PEPCK, phosphoenolpyruvate carboxykinase; TC, total cholesterol; TG, transgenic; WT, wild-type. assessed by hematoxylin and eosin staining and Oil Red $\mathrm{O}$ staining (Figure 4B). On OSMR $\beta$ deletion (OSMR $\beta$-KO) and RNA interference (AdshOSMR $\beta$ ), however, increased lipid droplet accumulation occurred (Figure 4B and Supplemental Figure S3H), which was correlated with a significant increase in hepatic triglycerides, TC, and NEFAs (Figure 4C and Supplemental Figure S3G). In sharp contrast, hepatic triglyceride, TC, and NEFA levels were largely restored in the OSMR $\beta$-TG mice (Figure 4C).

Consistent with a redirection in the glucose flux toward fatty acid synthesis, we observed that the OSMR $\beta$ expression level was negatively correlated with mRNA levels of gluconeogenic genes, including phosphoenolpyruvate carboxykinase and glucose-6-phosphatase in vivo (Figure 4D and Supplemental Figure S3I). Quantitative real-time PCR showed that OSMR $\beta$ ablation led to decreased levels of genes related to cholesterol efflux (eg, $A B C G 1)$ and fatty acid $\beta$-oxidation (eg, PPARA and CPTIA) in livers and increased expression levels of genes that regulate cholesterol synthesis (eg, SREBPlc), fatty acid synthesis (eg, ACCA, PPARG, and FAS), and uptake (eg, CD36 and $F A B P 1)$ compared with what was found in the livers of the WT or AdshRNA HFD controls (Figure 4E and Supplemental Figure S3J). In addition, OSMR $\beta$-KO mice also exhibited aggravated liver damage as evidenced by higher serum concentrations of liver aspartate aminotransferase and alanine aminotransferase (Supplemental Table S3). Accumulation of serum triglycerides, cholesterol, and free fatty acids was also more severe in OSMR $\beta$-KO mice (Supplemental Figure S4A). Notably, these adverse effects were reversed on liver-specific OSMR $\beta$ overexpression (Figure 4F and Supplemental Figure S4B; Supplemental Table S4).

Excessive lipid accumulation renders livers more vulnerable to adverse impacts, such as the presence of a proinflammatory mediator in the liver. ${ }^{25}$ Thus, we assessed whether OSMR $\beta$ regulates the hepatic inflammatory cytokine milieu in mice fed an HFD with the use of quantitative 


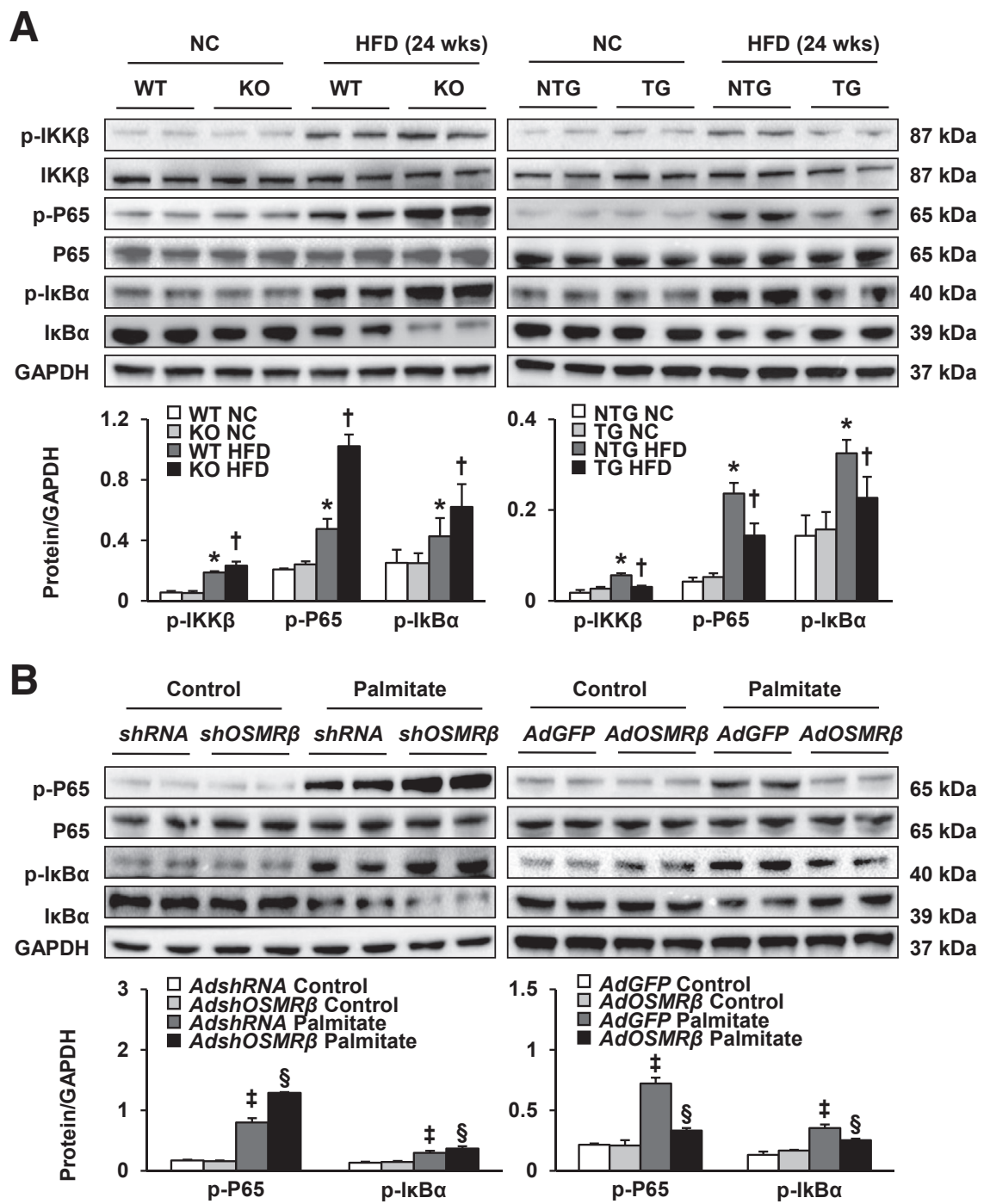

Figure 5 OSMR $\beta$ inhibits obesity-induced inflammation. A: Protein levels in the NF- $\kappa$ B signaling pathway in the livers were assessed using immunoblotting. B: Primary hepatocytes were infected with the indicated adenoviruses before challenging with $0.25 \mathrm{mmol} / \mathrm{L}$ palmitate. Immunoblotting and quantification of the NF- $\kappa \mathrm{B}$ signaling pathway were performed. Data are expressed as means \pm SD. $n=4$ per group (A and B). ${ }^{*} P<0.05$ versus WT or NTG NC group; ${ }^{\dagger} P<0.05$ versus WT or NTG HFD group; ${ }^{\ddagger} P<0.05$ versus AdshRNA or AdGFP control group; ${ }^{\S} P<0.05$ versus AdshRNA or AdGFP palmitate group. AdGFP, adenoviral vectors encoding the GFP gene; AdshRNA, adenoviral vectors encoding scrambled shRNA; GAPDH, glyceraldehyde-3-phosphate dehydrogenase; HFD, high-fat diet; KO, knockout; NC, normal chow; NTG, nontransgenic; OSMR $\beta$, OSM receptor $\beta$ chain; TG, transgenic; WT, wild-type. real-time PCR. The OSMR $\beta$-KO mice displayed higher levels of proinflammatory cytokines, including IL-1 $\beta$, IL-6, TNF- $\alpha$, MCP1, and inducible nitric oxide synthase, and lower levels of anti-inflammatory IL-10 compared with WT controls, whereas OSMR $\beta$-TG mice displayed an ameliorative inflammatory response (Supplemental Figure S4, C and D). Concomitantly, the serum concentrations of proinflammatory cytokines (eg, IL-1 $\beta$, IL-4, IL-6, TNF- $\alpha$, MCP1) were increased, and anti-inflammatory IL-10 was decreased on OSMR $\beta$ deletion (Supplemental Table S3), whereas OSMR $\beta$-TG mice displayed almost opposite expression concentrations of serum cytokines (Supplemental Table S4). Activation of the NF- $\mathrm{B}$ signaling pathway in the liver can be initiated by obesity and HFD, which leads to downstream cytokine production and subsequent hepatic inflammation. $^{26} \mathrm{We}$ observed that the phosphorylation levels of IKK $\beta$, p65, and I $\mathrm{B} \alpha$, which reflect the activity of $\mathrm{NF}-\kappa \mathrm{B}$, were significantly higher in the livers of OSMR $\beta$ $\mathrm{KO}$ mice but lower on OSMR $\beta$ overexpression compared with that of their respective controls (Figure 5A). The negative regulation of the $\mathrm{NF}-\kappa \mathrm{B}$ signaling pathway by OSMR $\beta$ was confirmed in primary hepatocytes in vitro (Figure 5B). Taken together, these data clearly demonstrate that OSMR $\beta$ protects against hepatic steatosis and inflammation in mice.

\section{Hepatic OSMR $\beta$ Overexpression Improves Metabolism} in the Genetic Obesity Model

To eliminate the confounding factors of HFD caused by unidentified components, we used a genetic obesity model to determine the biological effect of OSMR $\beta$ on hepatic metabolism. Leptin-deficient $(o b / o b)$ mice that had developed spontaneous hepatic steatosis ${ }^{24}$ were injected with adenovirus harboring OSMR $\beta$ (AdOSMR) through the jugular vein for liver-restricted OSMR $\beta$ overexpression and fed an $\mathrm{NC}$ diet for 4 weeks. Although the body weight remained comparable (Figure 6A), AdOSMR $\beta$ injections resulted in significantly reduced fasting serum glucose and insulin concentrations and a lower HOMA-IR index compared with that of 
A

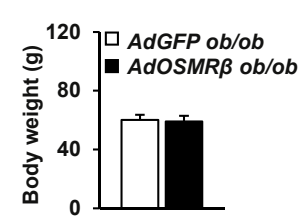

C

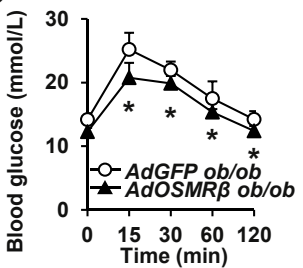

E

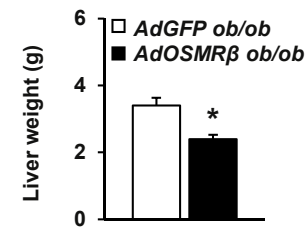

G

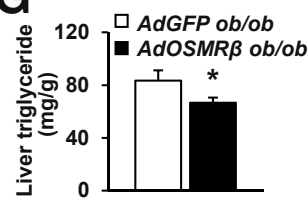

H

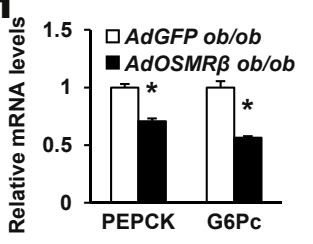

B
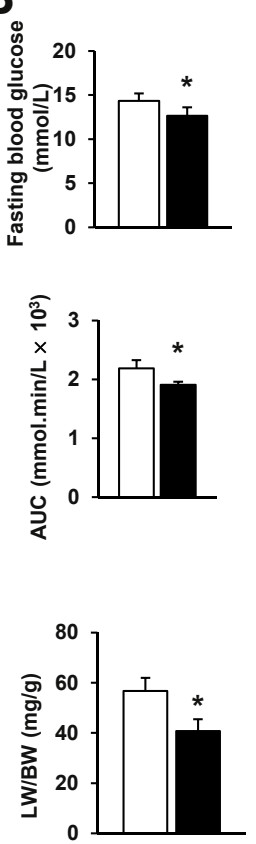

$F$

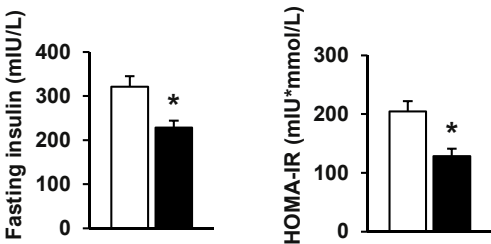

D
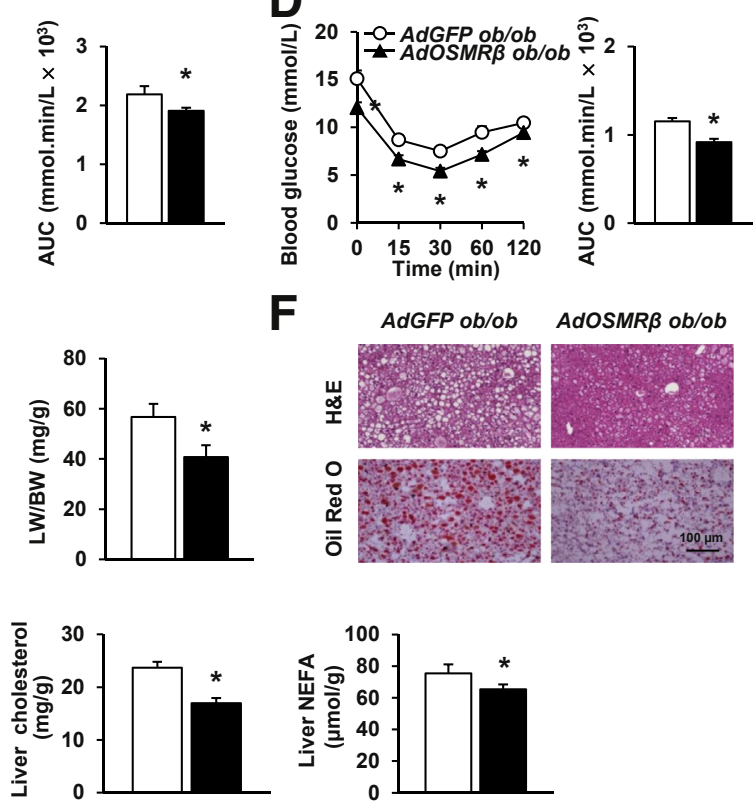

I

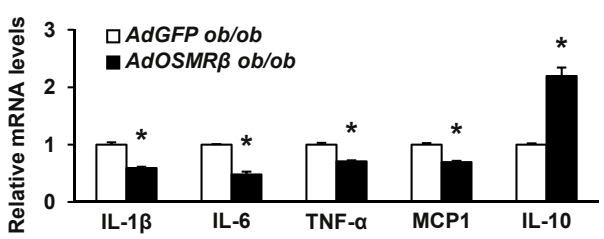

Figure 6 OSMR $\beta$ overexpression blunts hepatic insulin resistance, steatosis, and inflammation in $o b / o b$ mice. A: Body weight analysis of $o b / o b$ mice injected with AdOSMR $\beta$ or the AdGFP control. B: OSMR $\beta$ overexpression in the liver attenuates glucose homeostasis in the $o b / o b$ mice. Fasting serum glucose and insulin concentrations were detected at the indicated time points. HOMA-IR was also calculated. C and D: The IPGTT (C) and IPITT (D) assays were performed to evaluate the insulin sensitivity of mice in the indicated groups. E: Quantification of the body weights of the indicated mice and quantification of the LW/BW in the indicated group. F: Representative H\&E or Oil Red 0 staining of liver sections obtained from the AdGFP and AdOSMRB ob/ob groups. G: Levels of liver triglycerides, TC, and NEFAs were assessed. $\mathbf{H}$ : Quantification of mRNA levels of PEPCK and G6Pc using quantitative real-time PCR. I: Quantification of mRNA levels of proinflammatory and antiinflammatory markers in the liver. The error bars indicate the means \pm SD. $n=10$ to 14 per group (A); $n=8$ to 12 per group (B and $\mathbf{G}$ ); $n=8$ to 12 per group at each time point (C and $\mathbf{D}) ; n=8$ to 10 per group (I). ${ }^{*} P<0.05$ versus AdGFP ob/ob group. AdGFP, adenoviral vectors encoding the GFP gene; AdOSMR $\beta$, adenoviruses carrying sequences encoding mouse OSMR $\beta$; AUC, area under the curve; G6PC, glucose-6-phosphatase; HOMA-IR, homeostasis model assessment of insulin resistance; H\&E, hematoxylin and eosin; IPGTT, i.p. glucose tolerance test; IPITT, i.p. insulin tolerance test; LW/BW, ratio of liver weight to body weight; NEFA, nonesterified fatty acid; PEPCK, phosphoenolpyruvate carboxykinase; OSMR $\beta$, OSM receptor $\beta$ chain; TC, total cholesterol. adenovirus vectors encoding the GFP gene (AdGFP) controls (Figure 6B). Glucose regulation and insulin sensitivity were also improved on OSMR $\beta$ overexpression (Figure 6, $\mathrm{C}$ and D). Furthermore, OSMR $\beta$ overexpression ameliorated the established liver enlargement (Figure 6E) and hepatic lipid accumulation in $o b / o b$ mice (Figure $6 \mathrm{~F}$ ) and reduced hepatic triglyceride, TC, and NEFA content (Figure 6G). Moreover, gluconeogenesis was inhibited in AdOSMR $\beta$ mice compared with that of their AdGFP littermates (Figure 6H). We also observed significantly lower inflammatory responses in the livers of AdOSMR $\beta$-treated mice (Figure 6I). Thus, these data indicate that the administration of OSMR $\beta$ exerts beneficial effects on IR, hepatic steatosis, and inflammation in both diet-induced and genetic obesity.

\section{OSMR $\beta$ Activates the Hepatic JAK2/STAT3 Signal Transduction Pathway}

Several signaling pathways, including JAK2/STAT3 and MAPK, are stimulated by gp130 cytokines, although the spectrum of pathways that are activated varies, depending on the cell type and signaling effector. ${ }^{10}$ Interestingly, both pathways are critical regulators of IR and hepatic metabolism. 9,27 To explore how OSMR $\beta$ alleviates metabolic disorders, we harvested liver tissues from OSMR $\beta-\mathrm{KO}$ and OSMR $\beta$-TG mice fed an HFD for 24 weeks. Intriguingly, in vivo genetic manipulation did not affect phosphorylation of the MAPK family members, which exhibited unaffected activity (Supplemental Figure S5A). Nevertheless, the expression levels of phosphorylated JAK2 and STAT3, which are crucial for the maintenance of glucose homeostasis, were significantly reduced on OSMR $\beta$ deletion and knockdown compared with that of the respective controls (Figure 7A and Supplemental Figure S5B). Conversely, liver-specific overexpression of OSMR $\beta$ in both diet-induced and genetic obesity models resulted in significant phosphorylation and activation of JAK2/STAT3 signaling (Figure 7A and Supplemental Figure S5B). The positive regulation of the JAK2/STAT3 cascade by OSMR $\beta$ was also observed in primary hepatocytes challenged with palmitate (Figure 7B). These data suggest that OSMR $\beta$ activates the JAK2/STAT3 cascade but not the MAPK signaling pathway in the liver of obese mice. 


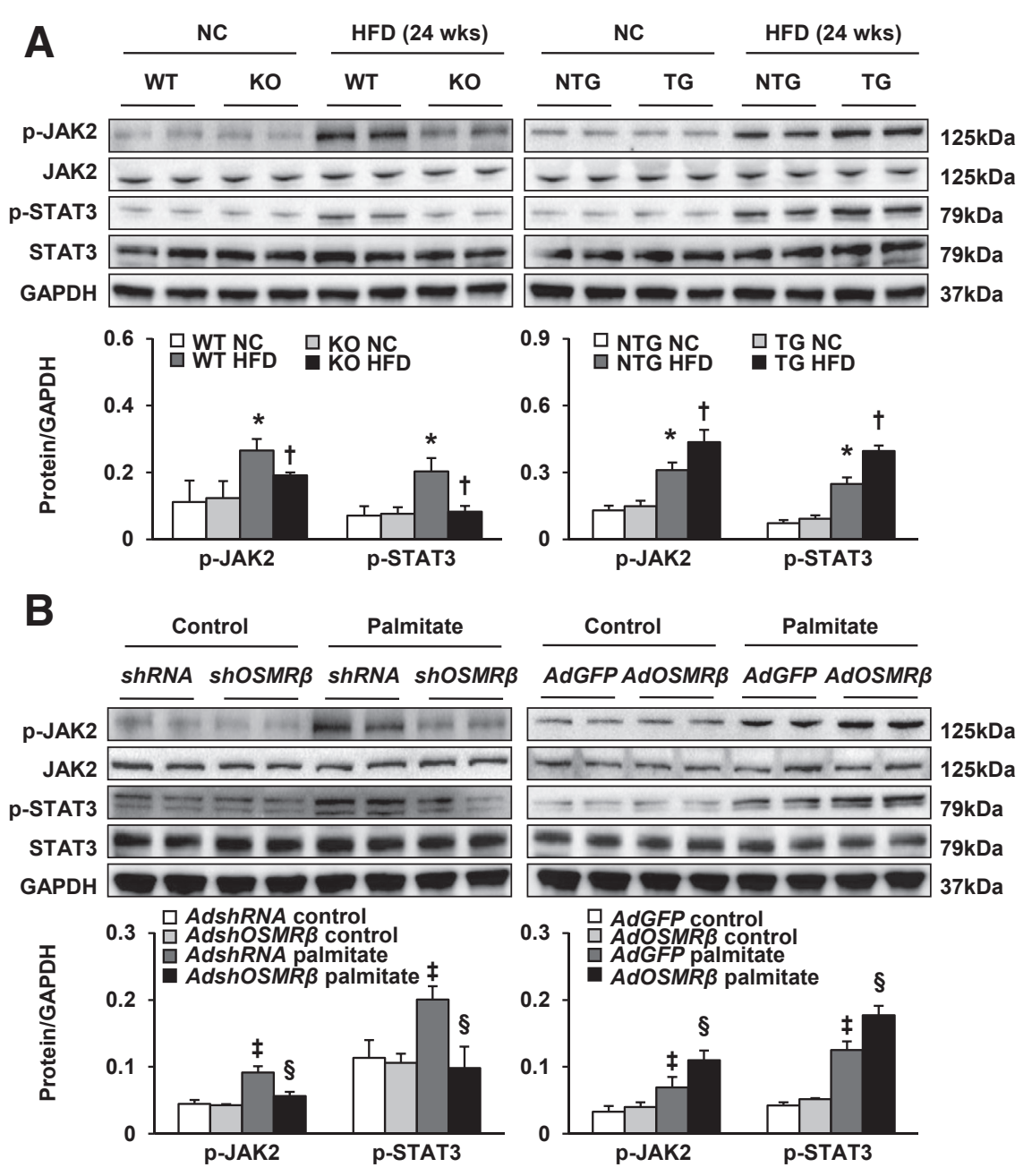

Figure 7 OSMR $\beta$ activates the JAK2/STAT3 pathway in liver. A: Protein levels of total and phosphorylated JAK2 and STAT3 were detected in the mouse livers by immunoblotting. GAPDH was used as a loading control. B: Immunoblotting was performed on primary hepatocytes infected with AdOSMR $\beta$ or AdshOSMR $\beta$ and subjected to 0.25 $\mathrm{mmol} / \mathrm{L}$ palmitate or the control treatment. Data are expressed as means \pm SD. $n=4$ per group. ${ }^{*} P<0.05$ versus WT NC or NTG NC; ${ }^{\dagger} P<0.05$ versus WT HFD or NTG HFD; ${ }^{\ddagger} P<0.05$ versus AdshRNA or AdGFP control group; ${ }^{\S} P<0.05$ versus AdshRNA or AdGFP palmitate group. AdOSMR $\beta$, adenoviruses carrying sequences encoding mouse OSMR $\beta$; AdshOSMR $\beta$, adenoviruses carrying a shRNA targeting OSMR $\beta$; GAPDH, glyceraldehyde3-phosphate dehydrogenase; HFD, high-fat diet; JAK2, Janus kinase 2; K0, knockout; NC, normal chow; NTG, nontransgenic; OSMR $\beta$, OSM receptor $\beta$ chain; TG, transgenic; WT, wild-type.

OSMR $\beta$ Ameliorates Glucose Tolerance, Hepatic Steatosis, and Inflammation through Activation of JAK2/STAT3 Signaling

Because OSMR $\beta$ promotes hepatic STAT3 phosphorylation in obese mice, we hypothesized that the beneficial role of OSMR $\beta$ in glucose homeostasis and hepatic steatosis may be attributable to STAT3 activation in the liver. To address this issue, we first examined whether STAT3 exerts similar protective effects in the absence of OSMR $\beta$. Liver-specific STAT3 overexpression transgenic mice, designated AlbSTAT3-TG mice, were generated with the Cre/LoxP system (Supplemental Figure S6A). Alb-STAT3-TG mice were then crossed with OSMR $\beta$-KO mice to generate OKST mice (Supplemental Figure S6B), which were validated via Western blot analysis (Supplemental Figure S6C). As expected, STAT3-TG mice displayed reduced body weight and improved glucose tolerance compared with control mice (Figure 8, A-D). More importantly, the deleterious effects of OSMR $\beta$ ablation were completely abrogated in the presence of joint STAT3 overexpression as evidenced by the comparable body weights (Figure 8A), fasting serum glucose and insulin (Figure 8B), and insulin tolerance (Figure 8, C and D) between the STAT3-TG and OKST mice. Moreover, STAT3 overexpression completely abolished liver enlargement (Figure 8E), lipid accumulation (Figure 8F), hepatic metabolic disorders (Figure 8G and Supplemental Figure S6D), and salient inflammation (Supplemental Figure S6E) observed in the OSMR $\beta-\mathrm{KO}$ mice. Thus, STAT3 is a main downstream target of OSMR $\beta$ in obesity-related hepatic steatosis and IR.

Subsequently, we determined whether OSMR $\beta$ could preserve its protective biological functions when JAK2/ STAT3 signaling was blocked. Therefore, we crossed OSMR $\beta$-TG mice with liver-specific STAT3-KO mice to generate OTSK mice (Supplemental Figure S7A). The overexpression of OSMR $\beta$ and deficiency of STAT3 in the liver were confirmed via Western blot analysis (Supplemental Figure S7B). Notably, OSMR $\beta$ overexpression in the absence of STAT3 (OTSK mice) did not protect against hepatic steatosis, IR, and hepatic inflammation, which was observed in OSMR $\beta$-TG mice (Figure 9 and Supplemental Figure S7, C and D). Instead, OTSK mice displayed a similar adverse phenotype to that in the STAT3-KO mice fed an HFD. Together, these data demonstrate that OSMR $\beta$ protects against glucose tolerance, hepatic steatosis, and inflammation via JAK2/STAT3 signal activation in the liver. 
A

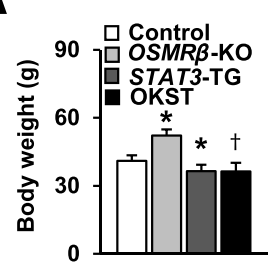

C

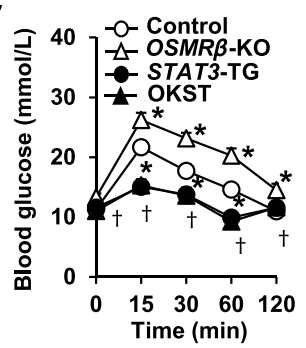

E

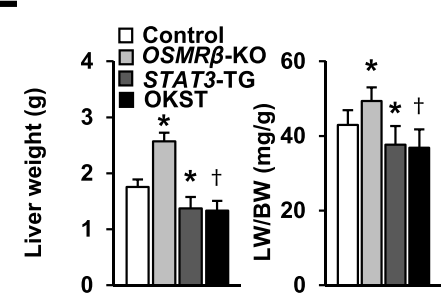

B
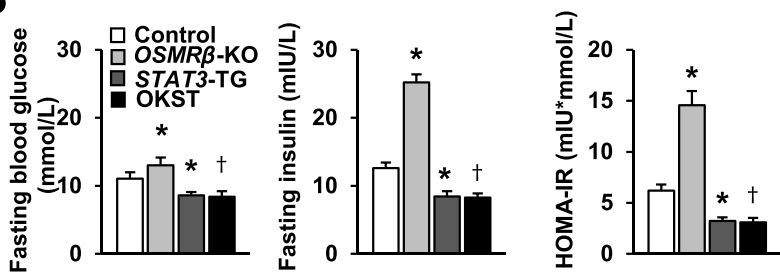

D
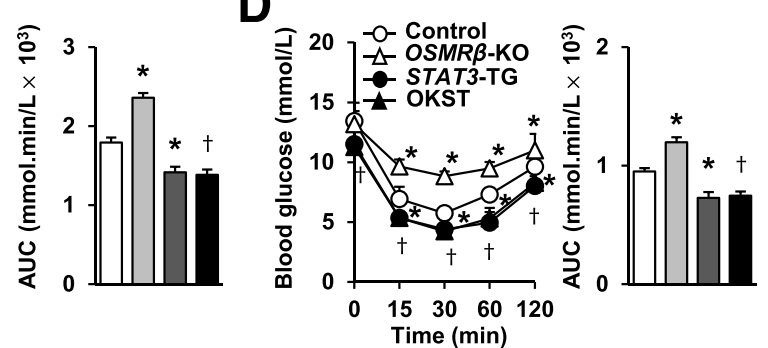

HFD

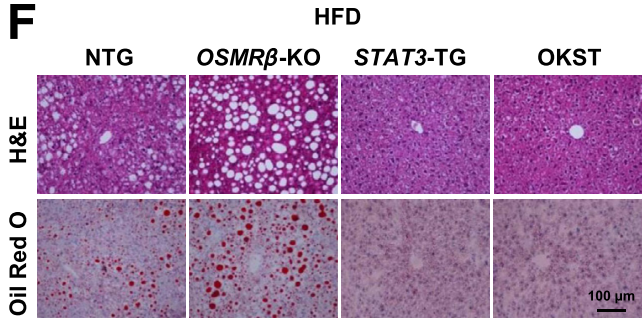

G
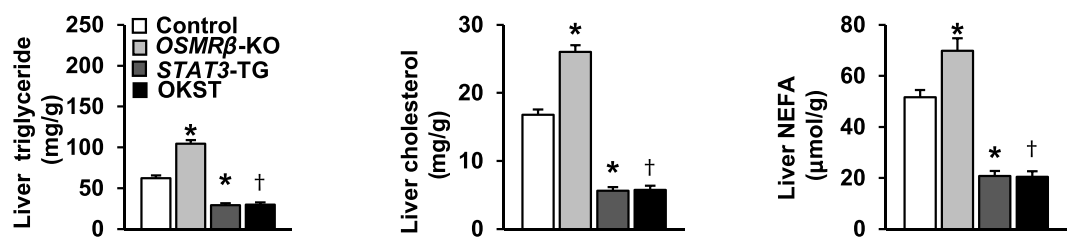

Figure 8 STAT3 overexpression in the liver mimics the protective effect of OSMR $\beta$. A: Analysis of the body weights of the OSMR $\beta-K 0$, STAT3-TG, and OKST mice fed an HFD. B: Fasting serum glucose and insulin concentrations are detected at the indicated time points, and HOMA-IR was calculated. C and D: Glucose tolerance was assessed by IPGTT (C) and IPITT (D). E: Quantification of the liver weight and ratio of liver weight to body weight in the indicated group. F: Representative H\&E or Oil Red 0 staining of liver sections. G: Levels of liver triglyceride, total cholesterol, and NEFAs were assessed. Data are expressed as means \pm SD. $n=9$ to 14 per group (A); $n=8$ to 12 per group (B); $n=8$ to 12 per group at each time point (C and $\mathbf{D}) ; n=9$ to 13 per group (E); $n=8$ to 10 per group (G). ${ }^{*} P<0.05$ versus control group; ${ }^{\dagger} P<0.05$ versus OSMR $\beta$-KO group. AUC, area under the curve; HFD, high-fat diet; HOMA-IR, homeostasis model assessment of insulin resistance; $\mathrm{H} \& \mathrm{E}$, hematoxylin and eosin; IPGTT, i.p. glucose tolerance test; IPITT, i.p. insulin tolerance test; $\mathrm{KO}$, knockout; $\mathrm{LW} / \mathrm{BW}$, ratio of liver weight to body weight; NEFA, nonesterified fatty acid; OKST, OSMR $\beta$-KO/STAT3-TG; OSMR $\beta$, OSM receptor $\beta$ chain; TG, transgenic.

\section{Discussion}

The liver is a key insulin-responsive organ responsible for insulin sensitivity and lipid metabolism. Recent studies have shown that OSMR $\beta$ is a crucial link in mediating adipose tissue inflammation and IR. ${ }^{19,20}$ However, the use of global $\mathrm{KO}$ mice in these studies increases the difficulty of determining the role of OSMR $\beta$ in specific tissues in the modulation of local and systemic inflammation and IR. In this study, we depicted the central role of hepatocyte-derived OSMR $\beta$ in the regulation of insulin sensitivity, hepatic steatosis, and inflammatory response for the first time. Several lines of evidence support this conclusion. First, OSMR $\beta$ was down-regulated in hepatic tissues in both human samples and mouse models of severe hepatic steatosis. Second, the whole-body deletion of OSMR $\beta$ aggravated HFD-induced metabolic disorders. Furthermore, liver-restricted OSMR $\beta$ knockdown with the use of an adenovirus-mediated gene-transfer approach confirmed the deteriorated results. In addition, we established liver-specific OSMR $\beta$ overexpression TG mice. Maintaining OSMR $\beta$ expression in the hepatocytes provided a beneficial effect on functional glucose tolerance and lipid metabolism. Third, OSMR $\beta$ phosphorylates and activates the JAK2/STAT3 signaling pathway in the liver. Finally, liver-restricted overexpression of STAT3 rescued glucose tolerance and ameliorated hepatic steatosis and inflammation in the OSMR $\beta$-KO mice. Conversely, OSMR $\beta$ overexpression failed to protect against hepatic steatosis, IR, and hepatic inflammation in the STAT3-deficient mice. We thus propose that hepatocytes are, at least partially, a crucial target for OSMR $\beta$-mediated beneficial effects on hepatic glucose homeostasis and lipid metabolism. Therefore, the targeted overexpression of OSMR $\beta$ in hepatocytes could be a novel therapeutic strategy against obesity-induced metabolic disorders.

OSMR $\beta$ expression is detectable in adipose tissue, primarily in adipose tissue macrophages and liver tissue. ${ }^{19,20}$ However, the distribution of OSMR $\beta$ in the liver, especially during the development of metabolic disorders, is less clear. In the current study, we observed that the protein expression of hepatic OSMR $\beta$ was significantly up-regulated in mice fed an HFD for 8 weeks, but dramatically downregulated in mice challenged with HFD for 16 and 24 
A

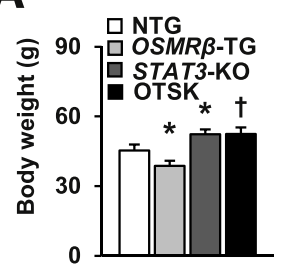

C

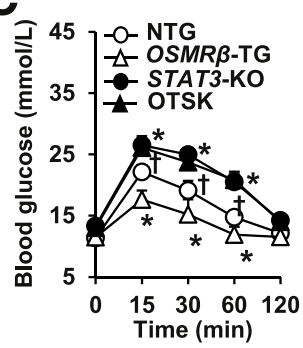

E
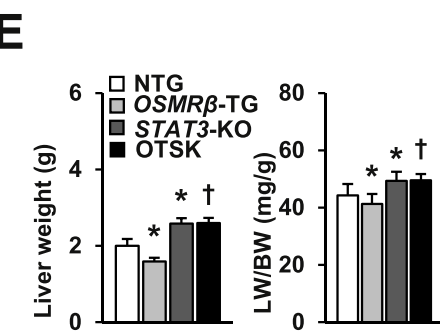

$\mathbf{F}$
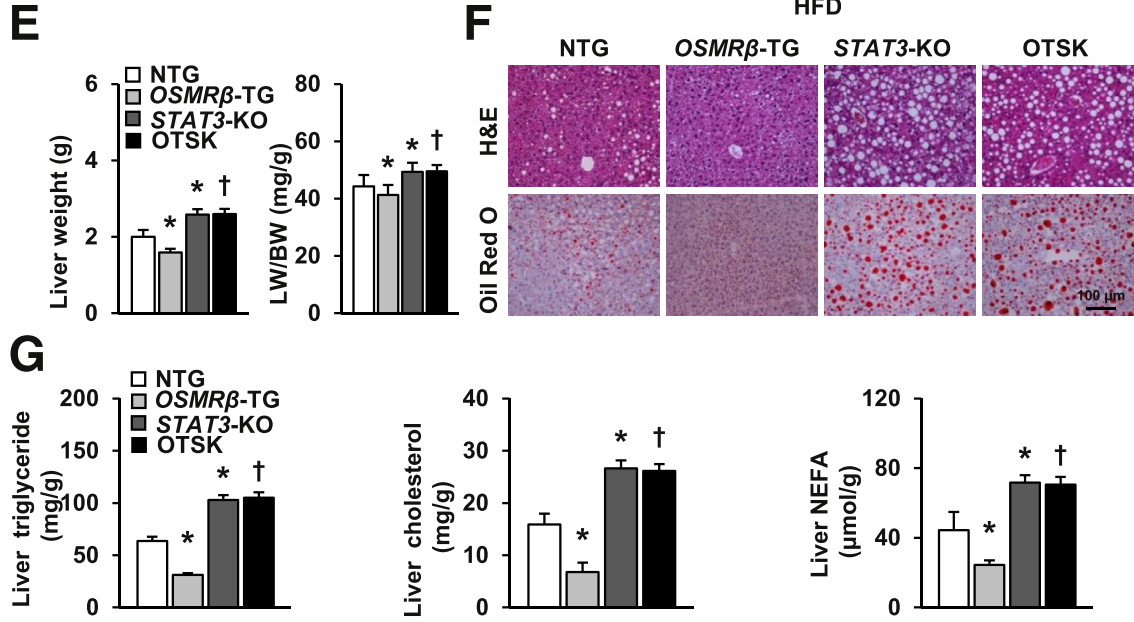

Figure 9 Metabolic protective effect of OSMR $\beta$ is STAT3 dependent. A: Analysis of the body weights of OSMRB-TG, STAT3-KO, and OTSK mice fed an HFD. B: Fasting serum glucose and insulin concentrations were detected at the indicated time points. HOMA-IR was also calculated. $\mathbf{C}$ and D: Glucose tolerance was assessed via IPGTT (C) and IPITT (D). E: Quantification of the liver weights and liver weight to body weight ratio in the indicated group. F: Representative H\&E or Oil Red 0 staining of liver sections. G: Levels of liver triglycerides, total cholesterol, and NEFAs were assessed. Data are expressed as means \pm SD. $n=$ 9 to 14 per group (A); $n=8$ to 12 per group (B); $n=8$ to 12 per group at each time point (C and D); $n=9$ to 14 per group (E); $n=8$ to 10 per group (G). ${ }^{*} P<0.05$ versus NTG group; ${ }^{\dagger} P<0.05$ versus OSMR $\beta$-TG group. AUC, area under the curve; HFD, high-fat diet; HOMA-IR, homeostasis model assessment of insulin resistance; H\&E, hematoxylin and eosin; IPGTT, i.p. glucose tolerance test; IPITT, i.p. insulin tolerance test; $\mathrm{KO}$, knockout; NEFA, nonesterified fatty acid; NTG, nontransgenic; OSMR $\beta$, OSM receptor $\beta$ chain; OTSK, OSMR $\beta$-TG/STAT3-KO; TG, transgenic. weeks. Consistently, a previous report showed that OSMR $\beta$ mRNA expression was elevated in the livers of mice treated with HFD for 8 weeks. ${ }^{19}$ Furthermore, we demonstrated that HFD induced persistent OSMR $\beta$ mRNA up-regulation between 8 and 24 weeks. Thus, the decreased protein levels of OSMR $\beta$ were possibly a result of degradation induced by post-translational modification. Consistent with our observation, OSM expression, which is up-regulated in both adipose and liver tissue in obese mice, has been shown to reduce the protein expression levels of OSMR $\beta$ in lung fibroblasts by ligand-induced receptor degradation. ${ }^{19,28}$ Future studies to determine the mechanism of post-transcription regulation of OSMR $\beta$ expression are required.

OSM engages either LIFR $\alpha$ or OSMR $\beta$ before recruiting gp130 and activating receptor signaling transduction. ${ }^{29}$ OSM is synthesized in Kupffer cells and macrophages in the liver and has paracrine-like effect on hepatocytes. ${ }^{30}$ Several of the observed metabolic alterations in OSMR $\beta$ $\mathrm{KO}$ mice are contrary to the known effect of OSM on hepatic metabolism. For example, Henkel et al ${ }^{18}$ showed that OSM produced by Kupffer cells attenuated insulindependent induction of Akt phosphorylation and glucokinase in rat primary hepatocytes and inhibited the expression of key enzymes of hepatic lipid metabolism, such as CPT-1. Notably, human OSM binds to only OSMR $\beta$ in mice, whereas human OSM recruits both OSMR $\beta$ and LIFR $\alpha$ in rats and humans. ${ }^{10,31,32}$ The current study investigated the functional role of OSMR $\beta$ in OSMR $\beta$-KO mice. We observed that OSMR $\beta$ positively regulates IRS1/Akt phosphorylation in obese mice. The use of OSMR $\beta$-KO mice in the current study eliminated the confounding effects of LIFR $\alpha$ in hepatic metabolism. Thus, the distinguishing difference observed between the rat hepatocyte model and OSMR $\beta$-KO mice may have been caused by the lack of interaction between OSM and the alternative LIFR $\alpha$ in OSMR $\beta-\mathrm{KO}$ mice. Consistent with our observations, Znoyko et $\mathrm{al}^{33}$ demonstrated that, although OSM was consistently expressed in cirrhotic human liver, OSMR $\beta$ expression was absent and LIFR $\alpha$ was up-regulated. Therefore, our data suggest that the metabolic function of OSM is attributable to speciesspecific ligand-receptor interactions, and targeting OSMR $\beta$ may be a more promising therapeutic strategy in humans than targeting OSM. 
Furthermore, we showed that liver-specific OSMR $\beta$ overexpression TG mice exhibited lower fasting serum glucose and insulin concentrations, improved insulin sensitivity, and attenuated hepatic inflammatory responses and dyslipidemia, further indicating that the liver is a crucial insulin-responsive organ responsible for insulin sensitivity and lipid metabolism. Mechanistically, OSMR $\beta$ expression positively regulates phosphorylation and activation of the downstream JAK2/STAT3 signaling pathway. In support of our data, Inoue et $\mathrm{al}^{9}$ showed that the liver-specific deletion of STAT3 resulted in enhanced IR associated with increased hepatic expression of gluconeogenic genes. We demonstrated that liver-restricted overexpression of STAT3 rescued glucose tolerance and ameliorated hepatic steatosis and inflammation in OSMR $\beta$-KO mice, whereas OSMR $\beta$ overexpression failed to protect against the adverse phenotypes in STAT3-deficient mice. Thus, we further extended these findings by demonstrating that STAT3 is both sufficient and required for OSMR $\beta$-mediated metabolic benefits. Moreover, STAT3 is also involved in hepatic ischemia/reperfusion injury, ${ }^{34}$ regeneration, ${ }^{35}$ and cancer, ${ }^{36}$ suggesting a more versatile role of OSMR $\beta$ in the liver.

Intriguingly, we observed that liver-specific overexpression of OSMR $\beta$ also led to enhanced insulin-induced AKT phosphorylation in adipose tissue and skeletal muscle, suggesting that hepatic OSMR $\beta$ is required for total body glucose tolerance and IR. Nevertheless, the precise mechanisms underlying the regulation of hepatic OSMR $\beta$ on total body insulin sensitivity needs further investigation. In addition, although we have shown that liver is a main target of OSMR $\beta$, adipose- and skeletal-specific gene manipulations of OSMR $\beta$ are required in future studies to determine the impact of local OSMR $\beta$ on IR in these tissues.

Our data demonstrate profound salubrious effects of OSMR $\beta$ in the setting of HFD administration. Of note, a previous study revealed that OSMR $\beta^{-1-}$ mice exhibited IR preceding obesity at 16 weeks of age, suggesting that OSMR $\beta$ also plays a vital role in maintaining metabolic homeostasis in lean mice. ${ }^{20}$ However, in the current study, gene manipulations of OSMR $\beta$ showed no significant effect on IR, hepatic steatosis, and inflammatory response when fed nromal chow. Indeed, overexpression of OSMR $\beta$ also failed to activate JAK2/STAT3 signaling pathway in lean mice as that observed in $o b / o b$ mice (Figure $6 \mathrm{C}$ ). We also observed a similar negative result in primary hepatocytes without palmitate challenge. Possible explanations for these discrepancies are the result of the difference in mice, diet composition, or others. Apart from the discrepancy, our results consistently demonstrated the crucial role of OSMR $\beta$ in maintaining metabolic homeostasis.

Taken together, our study suggests that OSMR $\beta$ expressed by hepatocytes plays a critical role in regulating obesity-induced metabolic disorders, including IR, hepatic steatosis, and inflammatory response. Furthermore, we demonstrated that OSMR $\beta$-mediated protection is largely
STAT3 dependent, at least when comorbid with obesity. In this context, preventing the suppression of the OSMR $\beta /$ STAT3 signaling pathway in hepatocytes could be a new strategy for attenuating metabolic disorders.

\section{Supplemental Data}

Supplemental material for this article can be found at http://dx.doi.org/10.1016/j.ajpath.2015.12.028.

\section{References}

1. Perry RJ, Samuel VT, Petersen KF, Shulman GI: The role of hepatic lipids in hepatic insulin resistance and type 2 diabetes. Nature 2014, 510:84-91

2. Birkenfeld AL, Shulman GI: Nonalcoholic fatty liver disease, hepatic insulin resistance, and type 2 diabetes. Hepatology 2014, 59:713-723

3. Glass CK, Olefsky JM: Inflammation and lipid signaling in the etiology of insulin resistance. Cell Metab 2012, 15:635-645

4. Samuel VT, Shulman GI: Mechanisms for insulin resistance: common threads and missing links. Cell 2012, 148:852-871

5. Berlanga A, Guiu-Jurado E, Porras JA, Auguet T: Molecular pathways in non-alcoholic fatty liver disease. Clin Exp Gastroenterol 2014, 7:221-239

6. Yoon HJ, Cha BS: Pathogenesis and therapeutic approaches for nonalcoholic fatty liver disease. World J Hepatol 2014, 6:800-811

7. Wang XA, Zhang R, Jiang D, Deng W, Zhang S, Deng S, Zhong J, Wang T, Zhu LH, Yang L, Hong S, Guo S, Chen K, Zhang XF, She Z, Chen Y, Yang Q, Zhang XD, Li H: Interferon regulatory factor 9 protects against hepatic insulin resistance and steatosis in male mice. Hepatology 2013, 58:603-616

8. Wang XA, Zhang R, She ZG, Zhang XF, Jiang DS, Wang T, Gao L, Deng W, Zhang SM, Zhu LH, Guo S, Chen K, Zhang XD, Liu DP, $\mathrm{Li} \mathrm{H}$ : Interferon regulatory factor 3 constrains IKKbeta/NF-kappaB signaling to alleviate hepatic steatosis and insulin resistance. Hepatology 2014, 59:870-885

9. Inoue $\mathrm{H}$, Ogawa W, Ozaki M, Haga S, Matsumoto M, Furukawa K, Hashimoto N, Kido Y, Mori T, Sakaue H, Teshigawara K, Jin S, Iguchi H, Hiramatsu R, LeRoith D, Takeda K, Akira S, Kasuga M: Role of STAT-3 in regulation of hepatic gluconeogenic genes and carbohydrate metabolism in vivo. Nat Med 2004, 10:168-174

10. Richards CD: The enigmatic cytokine oncostatin $\mathrm{m}$ and roles in disease. ISRN Inflamm 2013, 2013:512103

11. Richards CD, Brown TJ, Shoyab M, Baumann H, Gauldie J: Recombinant oncostatin M stimulates the production of acute phase proteins in HepG2 cells and rat primary hepatocytes in vitro. J Immunol 1992, 148:1731-1736

12. Chung B, Verdier F, Matak P, Deschemin JC, Mayeux P, Vaulont S: Oncostatin $\mathrm{M}$ is a potent inducer of hepcidin, the iron regulatory hormone. FASEB J 2010, 24:2093-2103

13. Miyajima A, Kinoshita T, Tanaka M, Kamiya A, Mukouyama Y, Hara T: Role of Oncostatin M in hematopoiesis and liver development. Cytokine Growth Factor Rev 2000, 11:177-183

14. Yamashita T, Honda M, Nio K, Nakamoto Y, Takamura H, Tani T, Zen Y, Kaneko S: Oncostatin $\mathrm{m}$ renders epithelial cell adhesion molecule-positive liver cancer stem cells sensitive to 5 -Fluorouracil by inducing hepatocytic differentiation. Cancer Res 2010, 70:4687-4697

15. Han MS, Jung DY, Morel C, Lakhani SA, Kim JK, Flavell RA, Davis RJ: JNK expression by macrophages promotes obesity-induced insulin resistance and inflammation. Science 2013, 339:218-222

16. Priceman SJ, Kujawski M, Shen S, Cherryholmes GA, Lee H, Zhang C, Kruper L, Mortimer J, Jove R, Riggs AD, Yu H: Regulation of adipose tissue $\mathrm{T}$ cell subsets by Stat 3 is crucial for diet-induced obesity and insulin resistance. Proc Natl Acad Sci U S A 2013, 110: 13079-13084 
17. Grove RI, Mazzucco CE, Radka SF, Shoyab M, Kiener PA: Oncostatin M up-regulates low density lipoprotein receptors in HepG2 cells by a novel mechanism. J Biol Chem 1991, 266: 18194-18199

18. Henkel J, Gartner D, Dorn C, Hellerbrand C, Schanze N, Elz SR, Puschel GP: Oncostatin M produced in Kupffer cells in response to PGE2: possible contributor to hepatic insulin resistance and steatosis. Lab Invest 2011, 91:1107-1117

19. Komori T, Tanaka M, Senba E, Miyajima A, Morikawa Y: Deficiency of oncostatin M receptor beta (OSMRbeta) exacerbates high-fat dietinduced obesity and related metabolic disorders in mice. J Biol Chem 2014, 289:13821-13837

20. Komori T, Tanaka M, Senba E, Miyajima A, Morikawa Y: Lack of oncostatin $\mathrm{M}$ receptor beta leads to adipose tissue inflammation and insulin resistance by switching macrophage phenotype. J Biol Chem 2013, 288:21861-21875

21. Li M, Feng B, Wang L, Guo S, Zhang P, Gong J, Zhang Y, Zheng A, Li H: Tollip is a critical mediator of cerebral ischaemia/reperfusion injury. J Pathol 2015, 237:249-262

22. Gomez-Lechon MJ, Donato MT, Martinez-Romero A, Jimenez N, Castell JV, O'Connor JE: A human hepatocellular in vitro model to investigate steatosis. Chem Biol Interact 2007, 165:106-116

23. Zhu LH, Wang A, Luo $P$, Wang $X$, Jiang DS, Deng W, Zhang X, Wang T, Liu Y, Gao L, Zhang S, Zhang J, Li H: Mindin/Spondin 2 inhibits hepatic steatosis, insulin resistance, and obesity via interaction with peroxisome proliferator-activated receptor alpha in mice. J Hepatol 2014, 60:1046-1054

24. Hebbard L, George J: Animal models of nonalcoholic fatty liver disease. Nat Rev Gastroenterol Hepatol 2011, 8:35-44

25. Tilg H, Moschen AR: Evolution of inflammation in nonalcoholic fatty liver disease: the multiple parallel hits hypothesis. Hepatology 2010, 52:1836-1846

26. Cai D, Yuan M, Frantz DF, Melendez PA, Hansen L, Lee J, Shoelson SE: Local and systemic insulin resistance resulting from hepatic activation of IKK-beta and NF-kappaB. Nat Med 2005, 11: $183-190$

27. Gual P, Le Marchand-Brustel Y, Tanti JF: Positive and negative regulation of insulin signaling through IRS-1 phosphorylation. Biochimie 2005, 87:99-109

28. Blanchard F, Wang Y, Kinzie E, Duplomb L, Godard A, Baumann H Oncostatin M regulates the synthesis and turnover of gp130, leukemia inhibitory factor receptor alpha, and oncostatin $\mathrm{M}$ receptor beta by distinct mechanisms. J Biol Chem 2001, 276:47038-47045

29. Caffarel MM, Coleman N: Oncostatin M receptor is a novel therapeutic target in cervical squamous cell carcinoma. J Pathol 2014, 232 : 386-390

30. Elks CM, Stephens JM: Oncostatin m modulation of lipid storage. Biology (Basel) 2015, 4:151-160

31. Chen SH, Benveniste EN: Oncostatin M: a pleiotropic cytokine in the central nervous system. Cytokine Growth Factor Rev 2004, 15: 379-391

32. Drechsler J, Grotzinger J, Hermanns HM: Characterization of the rat oncostatin $\mathrm{M}$ receptor complex which resembles the human, but differs from the murine cytokine receptor. PLoS One 2012, 7:e43155

33. Znoyko I, Sohara N, Spicer SS, Trojanowska M, Reuben A: Expression of oncostatin $\mathrm{M}$ and its receptors in normal and cirrhotic human liver. J Hepatol 2005, 43:893-900

34. Matsumoto T, O'Malley K, Efron PA, Burger C, McAuliffe PF, Scumpia PO, Uchida T, Tschoeke SK, Fujita S, Moldawer LL, Hemming AW, Foley DP: Interleukin-6 and STAT3 protect the liver from hepatic ischemia and reperfusion injury during ischemic preconditioning. Surgery 2006, 140:793-802

35. Debonera F, Aldeguer X, Shen X, Gelman AE, Gao F, Que X, Greenbaum LE, Furth EE, Taub R, Olthoff KM: Activation of interleukin-6/STAT3 and liver regeneration following transplantation. J Surg Res 2001, 96:289-295

36. He G, Karin M: NF-kappaB and STAT3 - key players in liver inflammation and cancer. Cell Res 2011, 21:159-168 